\title{
Large-scale CFD simulations of the transitional and turbulent regime for the large human airways during rapid inhalation
}

\author{
Hadrien Calmet ${ }^{\mathrm{a}, *}$, Alberto M. Gambaruto ${ }^{\mathrm{a}}$, Alister J. Bates ${ }^{\mathrm{b}}$, Mariano \\ Vázquez $^{\mathrm{a}}$, Guillaume Houzeaux ${ }^{\mathrm{a}}$, Denis J. Doorly ${ }^{\mathrm{b}}$ \\ ${ }^{a}$ Barcelona Supercomputing Center (BSC-CNS), Department of Computer Applications in \\ Science and Engineering, Edificio Nexus II - Planta 3 C/ JORDI GIRONA, 2908034 \\ Barcelona, Spain \\ ${ }^{b}$ Imperial College London, Department of Aeronautics, Exhibition Road, London SW7 2AZ, \\ $U K$
}

\begin{abstract}
The dynamics of unsteady flow in the human large airways during a rapid inhalation were investigated using highly detailed large-scale computational fluid dynamics on a subject-specific geometry. The simulations were performed to resolve all the spatial and temporal scales of the flow, thanks to the use of massive computational resources. A highly parallel finite element code was used, running on two supercomputers, solving the transient incompressible Navier-Stokes equations on unstructured meshes. Given that the finest mesh contained 350 million elements, the study sets a precedent for large-scale simulations of the respiratory system, proposing an analysis strategy for mean flow, fluctuations and wall shear stresses on a rapid and short inhalation (a so-called sniff). The geometry used encompasses the exterior face and the airways from the nasal cavity, through the trachea and up to the third lung bifurcation; it was derived from a contrast-enhanced computed tomography (CT) scan of a 48-year-old male. The transient inflow produces complex flows over a wide range of Reynolds numbers (Re). Thanks to the high fidelity simulations, many features involving the flow transition were observed, with the level of turbulence clearly higher in the throat
\end{abstract}

\footnotetext{
* Principal corresponding author

Email address: hadrien.calmet@bsc.es (Hadrien Calmet)
} 
than in the nose. Spectral analysis revealed turbulent characteristics persisting downstream of the glottis, and were captured even with a medium mesh resolution. However a fine mesh resolution was found necessary in the nasal cavity to observe transitional features. This work indicates the potential of large-scale simulations to further understanding of airway physiological mechanics, which is essential to guide clinical diagnosis; better understanding of the flow also has implications for the design of interventions such as aerosol drug delivery.

Keywords: CFD, airways, turbulence, inspiratory flow, respiratory airflow

\section{Introduction}

The flow in the human large airways is rich with complexity and variety. The geometry is extraordinarily complex with constriction zones and rapid changes in direction. Accordingly, a wide range of local Reynolds numbers (Re) occurs in the human large airways, which involve a mixture of various fluid motions. Further complications arise due to the fact that the breathing pace can change dramatically: at rest, whilst smelling or under heavy exercise. Previous studies state than while one is resting, flow is quasi-steady and laminar (see Zhao et al. [1]). On the other hand, during the kind of inspiration typical of a sniff, which is a rapid and short inhalation, the assumption of laminar flow is not justified. With the high amplitude of the inhalation and the rapid acceleration occurring during the sniff, the flow is transitional and turbulent.

Considering all these issues, the use of large-scale simulations becomes a must, as shown in several previous works where geometry and flow complexity are clearly exposed as core difficulties.

Doorly et al. [2] investigated variations in the nasal airways and in the modeling of flow in this complex geometry, providing comparisons [3] with numerical and experimental models. Lintermann et al. [4] also investigated variations in nasal airways, although under the scope of the respiratory efficiency. They classified different nasal cavities into ability groups, which support the a priori decision process on surgical interventions. 
To ensure a realistic inspiration model and velocity profile at the naris, Taylor et al. [5] demonstrated that incorporating the external nose and face not only reproduces the physiological situation, but also from a computational point of view furnishes additional information, such as the spatial position of particles over time. Other works, such as Jayaraju et al. [6] and Ball et al. [7], used the upper human airway with an idealized geometry and presented results concerning mean flow and flow structures. Contrary to Jayaraju et al. and Ball et al., Ghahramani et al. [8] used a realistic model to perform a numerical analysis in the upper human airway. Saksono et al. [9] and Lin et al. [10] demonstrated that incorporating the nasal cavity into the upper airway is essential in order to study the flow in the throat, since the results with and without the nasal cavity are dramatically different.

Many works have been performed with simplified models [11], idealized models $[6,7]$, or using several realistic models $[2,3]$, for example Choi et al. [12] investigated the variabilities of intra- and inter-subject of airflow in the human lung.

The laryngeal jet is the most important flow feature occurring in the throat during rapid inhalation. This phenomenon determines mean and fluctuating behaviors of the flow downstream in the tracheobronchiol airways [10]. The laryngeal jet involves various flow types, such as noncircular confined turbulent jet, open cavity flow, shear layers and curved and bifurcating turbulent and transitional pipe flow [12]. Choi et al. [12] detailed the unsteady behavior of the laryngeal jet through spectral analysis, Varghese et al. [13], described similar behaviors with the laryngeal jet with turbulence in a stenotic flow.

Another phenomenon known as flapping which is clearly an unsteady and transitional feature, may occur in the nasal cavity, i.e., in the anterior cavity $[2]$.

In the present paper, we focus on the sniff inspiration. A subject-specific model is used for this study, which extends from the face to the third branch generation of the bronchopulmonary tree (see Figures 1 and 2), created from a contrast-enhanced computed tomography (CT) scan of a 48-year-old male. 
The objective of this study is to determine the location of transition in the airways during a sniff using a realistic anatomical model and a high resolution of the computational mesh. The spatial and temporal resolutions in this study are very fine, in the order of $1 \mu \mathrm{s}$ for the temporal resolution and $0.1 \mathrm{~mm}$ for the spatial resolution throughout the domain; well beyond the resolution commonly applied in the literature today $[4,14]$.

This paper is organized as follows: section 2 presents the methods used, section 3 presents the results of the simulation, whilst the conclusion and the discussion are presented in Section 4.

\section{Numerical strategy}

To simulate the flow in the large human airways, computational meshes of different sizes were generated based on a single realistic geometry derived from Computer Tomography images. The numerical simulations were carried out using an in-house finite element code. In the following, brief descriptions of the geometry, the mesh, the fluid solver and the boundary conditions are given.

\subsection{Geometry}

The three-dimensional computational model was reconstructed from a clinically acquired computed tomography scan of a 48-year-old male subject, retrospectively collected from a large hospital database. The scan was performed in the supine position and the resulting data set comprises of 912 images in the axial plane, with $1 \mathrm{~mm}$ slice thickness and in-plane $0.65 \times 0.65 \mathrm{~mm}$ pixel size. Further details of the subject and acquisition are provided by Bates et al. [15]. Local ethics committee approval and patient consent were obtained to use the data for the investigation. A short description of the medical case is given in the appendix section.

The preliminary surface definition, obtained from a process of semi-automatic segmentation of the medical images, is of insufficient quality for direct use in numerical simulations. This is due to the limited resolution of the image stack 
together with the linear sub-pixel interpolation used in the segmentation, which results in an overly faceted and step-like surface representation. The resulting surface was then smoothed using Taubin's algorithm [16]. Surface mesh refinement was therefore performed, taking care that the refined surface definition lay within half a pixel size of the initial segmentation. Since this length scale is representative of the underlying uncertainty bounds, the resulting surface representation was deemed anatomically realistic and faithful to the medical data. A rigid-body reorientation to approximately align the posture to the Cartesian axes was performed as a final step to facilitate subsequent analysis.

\subsection{Mesh}

Meshing technique. The mesh discretisation used for scientific computing and simulation of the passages of the upper human respiratory system must be of high quality. An unstructured mesh was employed, due to the complex shape of the computational domain, especially apparent within the elaborate passageways of the nasal cavity. Given the importance of a good spatial discretisation, details of the mesh generation and size characteristics are outlined in the following. The mesh generation software employed was ANSYS ICEM CFD (ANSYS Inc., USA) and the description relates to the use of this software.

The approach used employs an octree-based method to generate a fine resolution surface mesh of the computational domain. Since this mesh resolution is finer than the previous representation, surface smoothing is once again required to avoid local flat patches and maintain a smoothly varying representation. The surface smoothing was performed using successive Laplace smoothing [17] and aspect ratio (ratio of shortest to longest edge) smoothing [18] in order to avoid surface shrinkage.

The second stage of the meshing process is the generation of a volumetric mesh, for which the Delaunay method was employed. This yielded a tetrahedral volume mesh with a smooth cell transition ratio $(\sim 1.2)$ employed close to the boundary wall. Finally a multi-layer prism mesh was created to resolve the high velocity gradients at the wall, with a few pyramids needed to ensure 


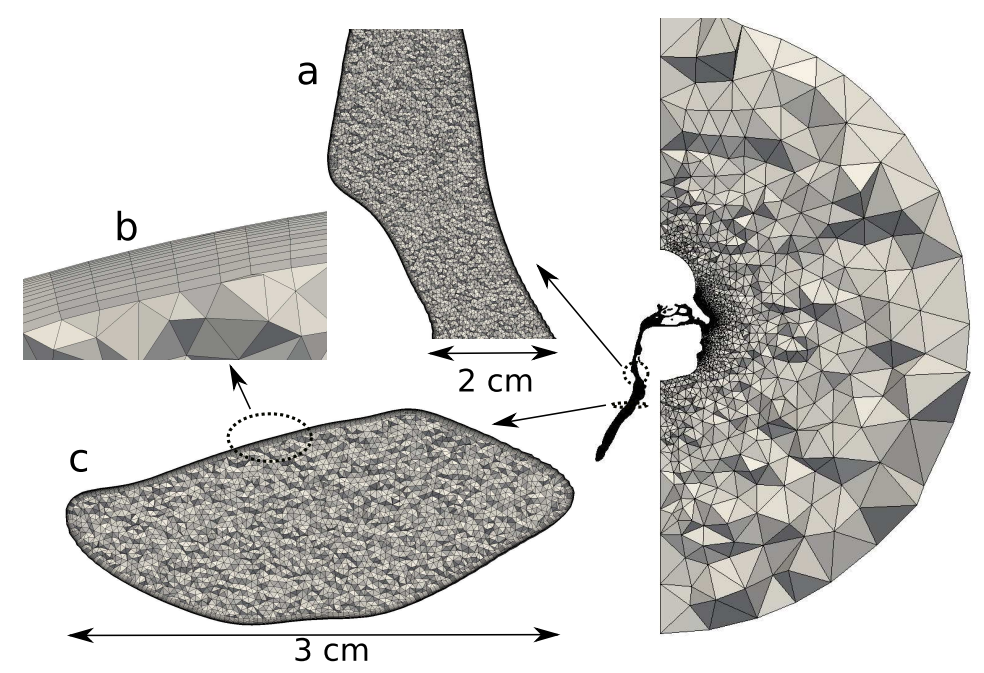

Figure 1: Sagittal view of upper airway mesh M2 (medium mesh) and details: (a) section in the sagittal plane above the glottis, (b) detail of prism layers, (c) section in the axial plane in the supra-glottic region.

the transition between the prism layer and tetrahedral mesh in the core of the computational domain.

Three meshes. Three different mesh sizes were created with the parameters given in Table 1.

\begin{tabular}{|l|c|c|c|c|c|c|c|}
\hline Mesh & $N_{N}\left(\times 10^{6}\right)$ & $N_{E}\left(\times 10^{6}\right)$ & $\Delta T(\mu s)$ & $h(m m)$ & $h_{b l 1}(\mu m)$ & $N_{b l}$ & $R_{b l}$ \\
\hline M1 (coarse) & 2 & 8 & 100 & 0.5 & 50 & 5 & 1.2 \\
\hline M2 (medium) & 14 & 44 & 10 & 0.3 & 6 & 13 & 1.2 \\
\hline M3 (fine) & 110 & 350 & 5 & 0.15 & 3 & 26 & 1.2 \\
\hline
\end{tabular}

Table 1: Summary of different mesh resolutions and simulation parameters with $N_{N}$ : number of nodes, $N_{E}$ : number of elements, $\Delta T$ : time step, $h$ : mean edge length of elements, $h_{b l 1}$ : height of the first element in the prism layer, $N_{b l}$ : number of prism layers and $R_{b l}$ : the prism growth ratio

The coarsest mesh (M1), with 8 million elements, was employed to obtain a general description of the flow field to identify regions requiring mesh refine- 
ment. The medium-sized mesh (M2), with 44 million elements, strikes a balance between computational costs and accuracy of solution, and was used to provide the majority of results presented in this study. Examples of the medium mesh (M2) are presented in Figure 1. For mesh M2, a minimum element height of $6 \mu \mathrm{m}$ at the wall in used for the most critical region (just above the glottis, see Figure 2), which corresponds to a value of $y^{+}=\frac{u_{*} y}{\nu}=0.5$ at the peak of the sniff inflow, where $u_{*}$ is the friction velocity at the wall, $y$ is the distance to the wall and $\nu$ is the local kinematic viscosity of the fluid. This value of $y^{+}$can be considered to be sufficiently small for resolving the near-wall flow dynamics [19] and falls into the range defined by Piomelli and Balaras [20] that describes well resolved wall-layers. The mesh generation of the coarse and medium meshes M1 and M2 was achieved using the ANSYS ICEM CFD sofware. Mesh M2 took 3 hours to be generated, on a single Intel Xeon X5650 core. A total of 4 GB of RAM was used while meshing.

The finest mesh (M3) was produced using the Mesh Multiplication technique described in [21]. This technique consists in refining the mesh uniformly, recursively, on-the-fly and in parallel, inside the simulation code. For tetrahedra, hexahedra and prisms, each level multiplies the number of elements by eight, while a pyramid is divided into ten new elements. Mesh M3 was obtained using a one-level mesh multiplication from mesh M2, therefore obtaining approximately (due to the presence of pyramids) eight times more elements. The time to produce this multiplied mesh in parallel is almost negligible [21].

\subsection{Fluid solver}

Discretization method. We consider the incompressible Navier-Stokes equations, one of the physical modules of the Alya system [22], a high performance computational mechanics code developed at BSC-CNS. The numerical model to solve these equations is based on a stabilized finite element method. The stabilization is based on the Variational MultiScale method which is considered as an implicit Large Eddy Simulation method [23]. The formulation is obtained by splitting the unknowns into grid scale and subgrid scale components. In the 
present formulation of Alya, the subgrid scale is, in addition, tracked in time and in space, thereby giving more accuracy and more stability to the numerical model [24].

Algebraic solver. The discretization of the Navier-Stokes equations yields a coupled algebraic system to be solved at each linearization step within a time loop. The resulting system is split to solve the momentum and continuity equations independently. This is achieved by applying an iterative strategy to solve for the Schur complement of the pressure. According to our scheme, at each linearization step it is necessary to solve the momentum and continuity equation twice. This split strategy is described and validated in [25].

The momentum equation algebraic system is solved using the GMRES method, with a simple diagonal preconditioner with a relatively low Krylov dimension. Usually, convergence is obtained in tens of iterations. The continuity equations is solved using the Deflated Conjugate Gradient (DCG) method [26], together with a linelet preconditioner to accelerate the convergence in the boundary layers [27].

Parallelization. The Alya code is written in Fortran90/95 and parallelized with MPI. The partition of the mesh is carried out on-the-fly with the METIS library [28]. The time for the partition is only a few seconds, even for mesh M3, for which the mesh multiplication is performed after the partition.

The fluid solver consists mainly of two basic steps, the assembly of the matrix and the right hand side of the Navier-Stokes system, and the solution of this system using the iterative solvers described previously. The assembly step does not require any communication, and its speedup is directly related to the load balance provided by METIS. The MPI exchanges in the iterative solvers are carried out in an asynchronous way, enabling to overlap work and communication during the matrix-vector products. The parallelization of the DCG solver is based on MPI gather functions, as extensively described in [26]. Finally, parallel $\mathrm{I} / \mathrm{O}$ is achieved using the HDF5 library [29] in order to postprocess the velocities 
and pressure on the mesh. In addition, filtering strategies enable to postprocess these variables on reduced portions of the mesh or to track single point values at each time step. These filtering techniques are necessary to reduce the amount of stored data and accelerate their visualizations.

\subsection{Boundary conditions}

The computational domain is extensive and comprises, in order of inspired flow direction, a hemisphere of the subject's face exterior, followed by the bilateral nasal passages, through to the pharynx, larynx, trachea and down to the third branch generation in the lungs, as shown in Figures 1 and 2. A no-slip boundary condition is imposed on the passage walls and the flat surface of the external hemisphere.

Inflow condition. The inflow velocity is imposed as a Dirichlet condition on the hemisphere dome. It is prescribed as a time varying uniform velocity with direction normal to the hemisphere. The radius of the hemisphere is $0.5 \mathrm{~m}$. It was chosen to be large enough to avoid the inlet boundary conditions affecting the flow field within the airways on expiration $(0.3 \mathrm{~m}$ and $1 \mathrm{~m}$ were also tested). Consequently, for inspiration this hemisphere size is considered to be sufficient. To model the sniff, we use a polynomial function of order 10 derived from the experimental work detailed in Rennie and al. [30], in which several short inhalations from rest as initial conditions were measured for different subjects. The $10^{\text {th }}$ order polynomial function (see Eq. 1) describing the temporal evolution of the flow rate is provided in Figure 3, with the coefficients of the flow defining equation 1 are referenced in the appendix section.

$$
P(x)=\sum_{i=0}^{10} C_{i} x^{i} \times 1.74
$$

Outflow condition. A zero-traction outflow condition is imposed as a Neumann condition (the surface is free from external stress) at the third bronchial branch. In order to ensure fully developed flow at the outflow sections, the final bronchial branches were extruded to constant cross-sectional pipes for several 


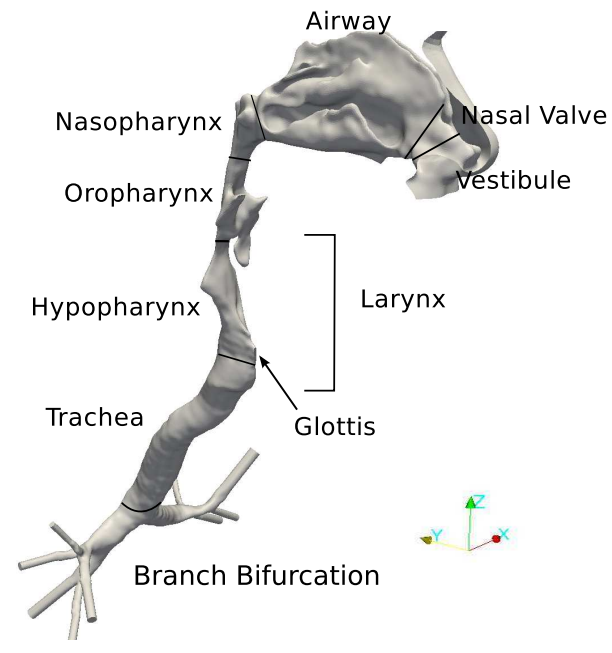

(a)

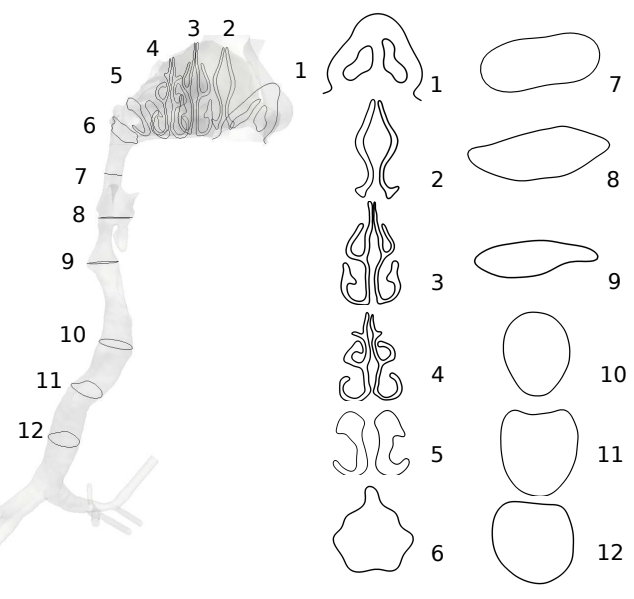

(b)

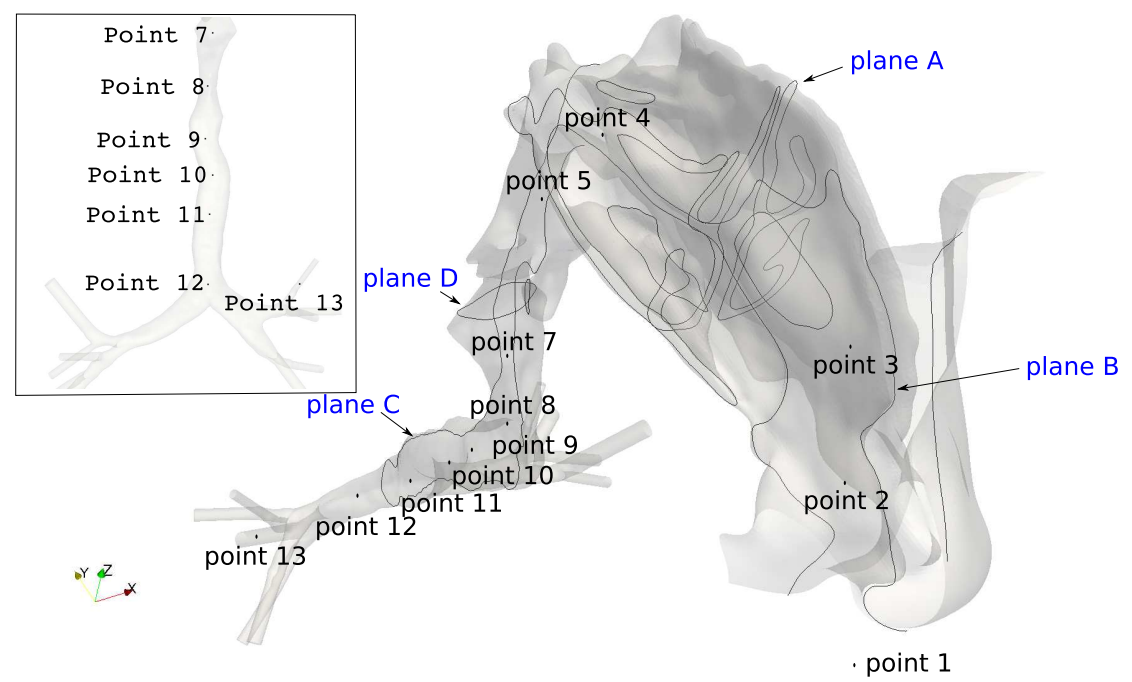

(c)

Figure 2: (a) Nomenclature of the approximate regions of the upper airways. (b) Location of different slices along the airways. (c) Locations of sections and points used as measurement locations for the results. Positions of planes within the airways are: plane A: coronal through nasal cavity, plane B: sagittal through right nasal cavity, plane C: sagittal through descending airways and plane D: coronal through supra-glottic region. Point coordinates are given in the appendix, see table 3. Plane A and slice 3 are the same. 


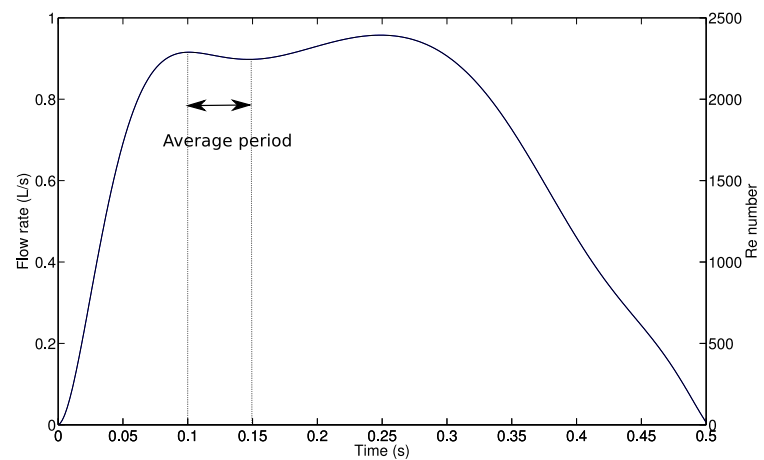

Figure 3: Flow rate profile with the local Reynolds number and average period, The $10^{t h}$ order polynomial function is given in the appendix see Equation 1 and Table 4. The local Reynolds number was calculated based on the area-average velocity and hydraulic diameter on the slice 1 located in the nasal valve (see figure $2 \mathrm{~b}$ ).

diameters. These bronchial branches are set to have the 5 last layers of elements with higher constant viscosity to reduce turbulence and thus to avoid entrant flow that would unstabilize the solution.

\subsection{Mesh convergence}

The mesh convergence study was performed by time averaging the velocity magnitude profile at a location in the trachea. The time average was taken over the period shown in Figure 3, during the plateau phase [0.1-0.13 s]. The velocity profile is shown over a line extending from the anterior to posterior along the middle of slice 11 , defined in figure $2 \mathrm{~b}$.

\subsection{Computational resources}

The simulations were carried out on the MareNostrum supercomputer, hosted by the Barcelona Supercomputing Center, and on the Fermi supercomputer, hosted by Cineca. For example, to carry out the simulation for the medium mesh M2 on the MareNostrum, 50000 time-steps are required on 1456 cores requiring approximately 200 hours. On the Fermi supercomputer, 16384 cores 


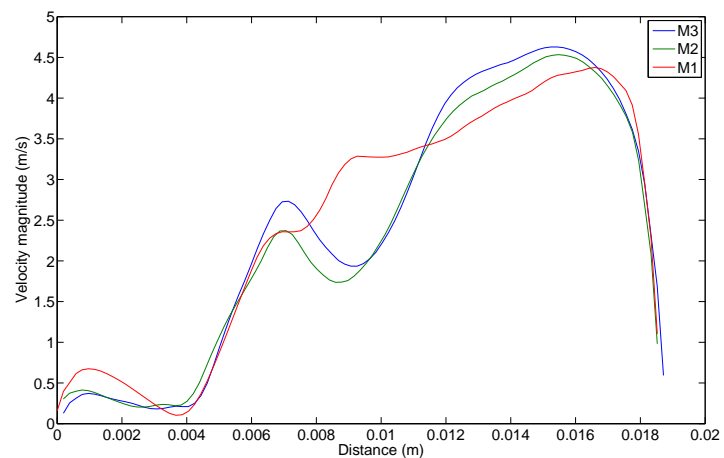

Figure 4: Time average velocity magnitude profile with different mesh resolution at the sagittal plane on the slice 11 , see figure $2 \mathrm{~b}$.

were used. Usually, in order to keep a good parallel efficiency, between 20000 and 40000 elements are used on each core. This makes the RAM requirement of the simulation very low under the possibilities of the computational nodes of the supercomputers used in this work.

\section{Results}

A sniff is a short, transient and unsteady inspiration, defined by the flow rate imposed as detailed in Section 2.4. The flow rate profile of the sniff is shown in Figure 3, and can be divided into three phases: the acceleration [0-0.1 s], the plateau [0.1-0.4 s] and the deceleration [0.4-0.5 s]. Each phase results in a distinct flow field, largely due to the resulting forces driving the inspiration. The acceleration phase is known to produce roll-up structures in the nasal cavity for a zero initial velocity [2], quickly establishing the bulk flow field due to the small cross-sectional passageways. Bates et al. [15] noted that nasal washout and stabilisation of nasal regional flow division occur within the first phase. The plateau phase is the most appropriate segment of the flow profile for the turbulence analysis as the velocity is approximately constant, and the time window is of sufficient duration to calculate turbulence statistics. The last phase is the deceleration, where breakup of flow structures and vortices begin 

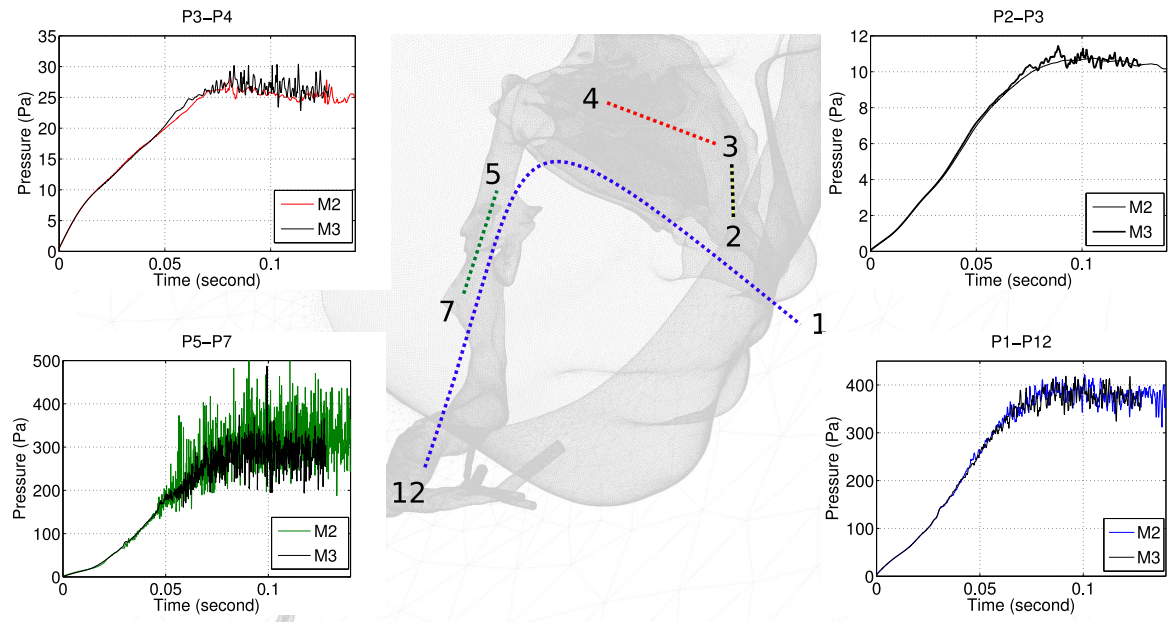

Figure 5: Static pressure drop across different compartments of the upper respiratory system during time period [0.0-0.13 s]. Comparison for mesh resolution M2 and M3 is shown.

to dominate. These are quickly suppressed in the nasal cavity due to the small patency of the passages. For more explanation of the three phases, see Bates et al. [15].

A time window during the plateau phase of $[0.1-0.15 \mathrm{~s}]$ is chosen to compute the mean flow and the turbulence measures. This window was taken as sufficiently long due to the fine temporal resolution available, and sufficiently short to avoid large-scale transients to interfere with the analysis. Also, the right nasal cavity was focused on, due to interesting features, such as recirculation in the anterior nasal cavity, which was not present in the left cavity of this subject for this flow profile but has been reported in other studies [2,31]. Unless otherwise stated, results are presented using the medium-sized mesh M2, except for the sniff beginning [0.0-0.13 s], where the M3 was used. This was due to temporary CPU usage limitations at the time of running the cases for this paper.

\subsection{Pressure drop}

Physiologically, airflow through the respiratory system is driven by the pressure drop, Figure 5 presents the static pressure drop across compartments of the 
airways. A comparison is made for the mesh resolution, M2 and M3. The results displayed are for the beginning of the sniff waveform, time period [0.0-0.13 s].

The pressure drops in the figure 5 highlight the role of the different compartments in the upper respiratory system:

- The pressure drop across the anterior nasal valve (P2-P3), i.e: between before the anterior nasal valve (point 2) and after the anterior nasal valve (point 3), in the right cavity. The anterior nasal valve is the smallest cross section area in the vestibule.

- The pressure drop across the nasal cavity (P3-P4), i.e: between after the nasal valve (point 3) and before the nasopharynx (point 4) in the right cavity. The nasal cavity is a very complex geometry with very thin cross sections. It is composed by three passageways, superior, middle and inferior meati.

- The pressure drop across the larynx (P5-P7), i.e: between the oropharynx (point 5) and after the larynx (point 7). This zone plays an important role in the turbulent flow in the descending airways.

- The overall pressure drop (P1-P12) i.e: between outside the nose (point 1) and the carina (at the first bifurcation of the lung) (point 12).

The values of pressure drop in Figure 5 show clearly that the highest pressure loss occurs in the throat and not in the nose. The pressure drop across the anterior nasal valve (P2-P3) is less than across the nasal cavity (P3-P4), despite it being a narrower section.

Oscillations are observed for the fine mesh (M3) in the anterior nasal valve pressure drop trace. This indicates that a fine mesh resolution $(0.15 \mathrm{~mm})$ is needed to capture features occurring in this region, as discussed later in Section 3.6. There are also higher amplitude of oscillations in the nasal cavity with the fine mesh solution, while in the remaining region the pressure drop appears sufficiently resolved. On the other hand in the larynx (P5-P7) there is 
a higher amplitude of oscillation and also a higher mean pressure drop value for the medium mesh (M2) compared to the fine one (M3).

The computed tomography (CT) scan is performed with the patient in the supine position. Gravity causes a collapse of the peripharyngeal tissues in the oropharynx, leading to a reduced patency; while in a standing posture the airspace in the larynx is greater. This observed collapse leads to the high pressure drop in the larynx (P5-P7).

\subsection{Mean flow}

The time window [0.1-0.15 s], used to compute the temporal mean velocity, is chosen as part of the plateau phase corresponding to the same time period for which the turbulence analysis is performed. The flow rate is approximately constant during this time period, and the mean velocity provides an overview of the dominant persistent flow features.

Figure 6 shows the mean velocity in four different cross sections of the airway, chosen to be approximate mid-passage locations. In the sagittal plane of the right nasal cavity (plane B) two recirculation areas are visible: the first is small and centred in the vestibule (B1), while the second is located in the upper anterior part of the nasal cavity (B2) and is both larger and more complex. The B2 recirculation region is caused by flow separation at the superior edge of the nasal valve. This feature has been observed in the literature, for instance in the experimental study of Doorly et al. [2] with the use of a high speed PIV setup, where it was stated that a shear layer that is generated on the boundary of the separation region, causes a transitional flow that is observed as periodic flapping (and will be discussed in greater detail in Section 3.6). The nasal valve has a small cross-sectional area and causes the flow to focus and form a jet that is directed towards the middle turbinate leading edge. A rapid change in geometry orthogonal to the jet direction occurs downstream of the nasal valve and is especially pronounced on the superior portion of the nasal valve. This superior portion is the thinnest section of the nasal valve [31], and is often related to corrective surgery to widen and increase the angle it forms. 

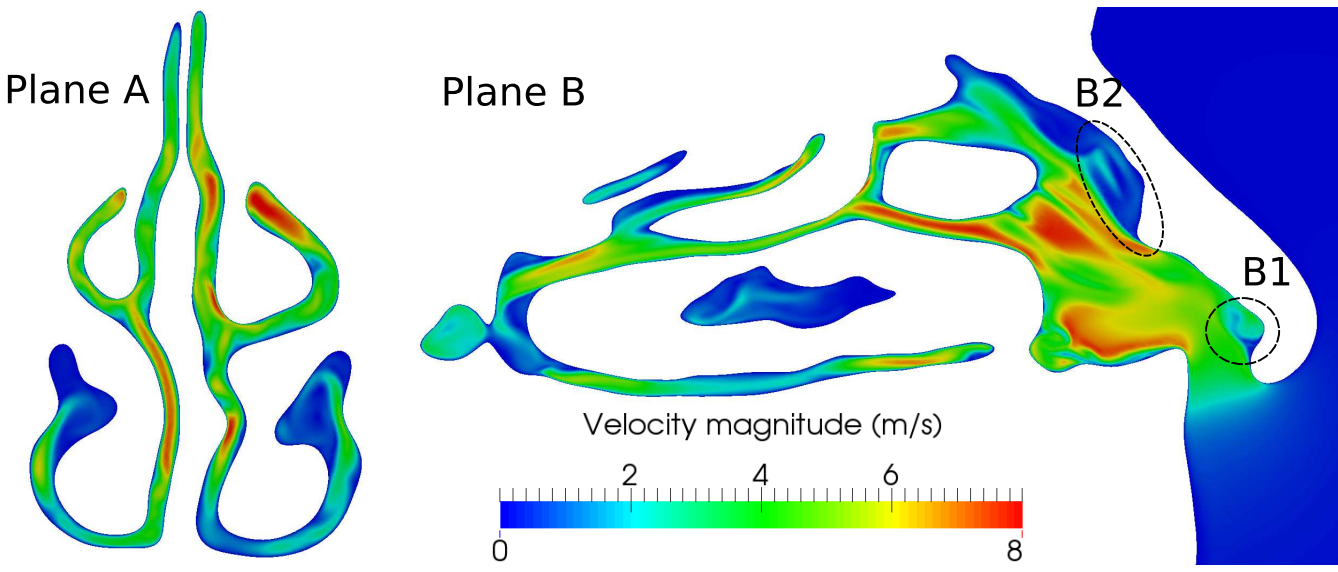

(a)

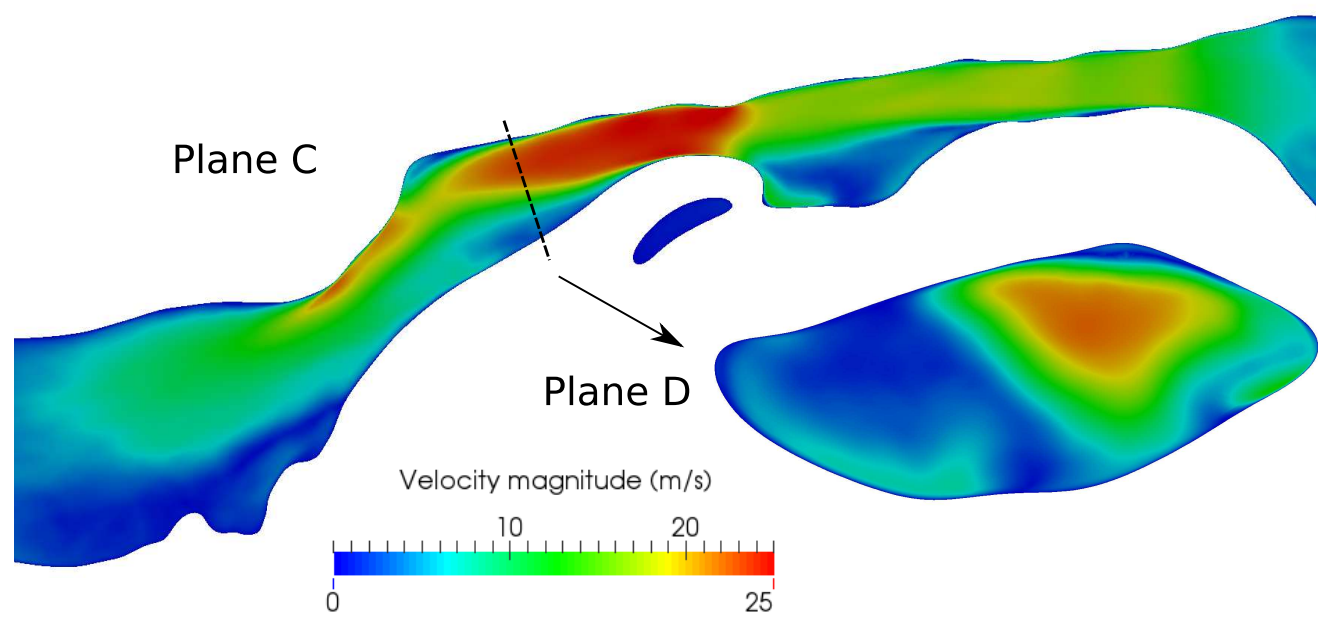

(b)

Figure 6: Mean velocity of cross-sections. (a) Coronal plane in the middle of nasal cavity (plane A) and sagittal plane of right nasal cavity (plane B) with the two recirculation zones. (b) Sagittal plane of laryngotracheal area (plane C) and a coronal plane of the supra-glottic region(plane D). (a) and (b) are computed for the time period [0.1-0.15 s] and the mean velocity scale is different to enhance the features in the nasal cavity and in the laryngotracheal area. See Figure 2 for cross-section location. 
The flow distribution in the nasal cavity can be observed by a coronal crosssection (see plane A), as this plane is perpendicular to the bulk flow direction. It is evident that the flow distribution between the bilateral passages is not the same due to evidently different passage shapes and patency. It can be observed however that the bulk of the flow inspired traverses the middle portion of the nasal cavity, leading to slow flow velocity in the lower meatuses despite their large dimensions. Higher flow rates are observed in the centrer-line of the septum and middle meatus passages. The olfactory region, located at the superior part of the nasal cavity, has a slower flow rate and is an indication that the transport phenomena are characterised by both advection and diffusion in this region. In comparison to slower flow rates however $[2,31]$, a sniff advects a larger volume of inspired air towards the olfactory cleft.

In the oropharynx region, there is another marked reduction in the passage cross-sectional area. This stenosis prompts a jet to be formed, as seen in planes $\mathrm{C}$ and $\mathrm{D}$. This jet has also been reported in the literature where it has been referred to as the laryngeal jet $[12,32]$. This jet persists a short distance downstream, as seen by a region of focused high magnitude velocity, and is broken down rapidly. The rapid breakdown is characterised by the short length and fast decrease with regard to the jet mean velocity, as the flow passes the larynx and enters the trachea. This indicates noticeable temporal fluctuations (low mean values), as will be discussed later in Section 3.3. As the passage changes direction, the jet impinges on the posterior hypopharynx wall and causes a high wall-shear stress region (as shown in Figure 10 for a single time instant), that together with the expanding passage area at the start of the trachea, promotes the jet breakup.

Iso-surfaces of mean velocity in the laryngotracheal region are shown in Figure 7 and provides a three-dimensional representation of the laryngeal jet. Two vortex structures are also identifiable at the upstream region to the oropharynx stenosis, and are the result of Dean vortices formed by the passage 90 degree change in angle at the nasopharynx. From the front view of Figure 7, it can be seen that the laryngeal jet is deviated downstream of the glottis, due to the 


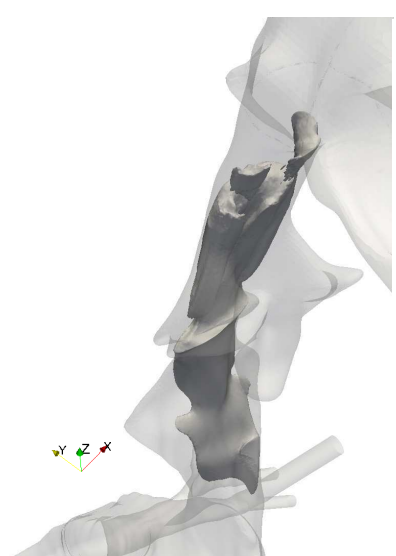

(a)

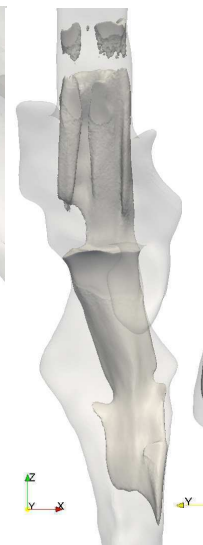

(b)

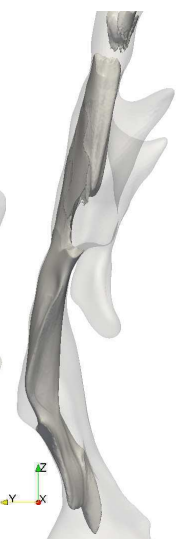

(c)

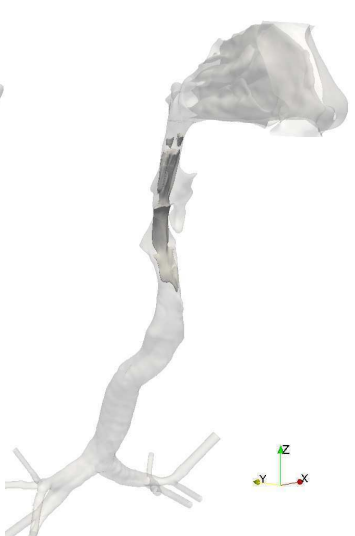

(d)

Figure 7: Different views of the laryngeal jet, given as the mean velocity iso-surface, computed for the time period [0.1-0.15 s]. (a) Top isometric view. (b) Front view. (c) Lateral view. (d) Isometric view.)

sinuous geometry related to the posture of the subject during the scan.

Figure 8 shows four different slices (described in Figure 2) with the time average velocity $\langle\mathbf{u}\rangle$ and the instantaneous velocity $\mathbf{u}$. It is apparent that the mean velocity does not differ significantly from the time instant one within the nasal passages, however in the descending tract a noticeable difference is evident.

\subsection{Fluctuations in the flow}

Having outlined the flow features discernible from the mean flow field, we now turn our attention to the temporal fluctuations. The root mean squared velocity fluctuations vector is computed for each component of the velocity vector like $u_{r m s}^{\prime}=\sqrt{\left\langle u^{\prime 2}\right\rangle}$, for the time period [0.1-0.15 s]. The resulting vector magnitude, presented in Figure 9 for some cross-sections of the flow field, can highlight the unsteady flow regions with respect to the mean flow.

An immediate result of the $\left|\mathbf{u}_{r m s}^{\prime}\right|$ is that in the nasal cavity, the magnitude of the fluctuations are an order of magnitude smaller than in the laryngeal region. Similar results have been reported in Zhao et al. [1], which noted that the airflow in the human nasal cavity even during sniffing is transitional or exhibits low intensity turbulence. Bates et al. [15] stated that, for the same subject used 


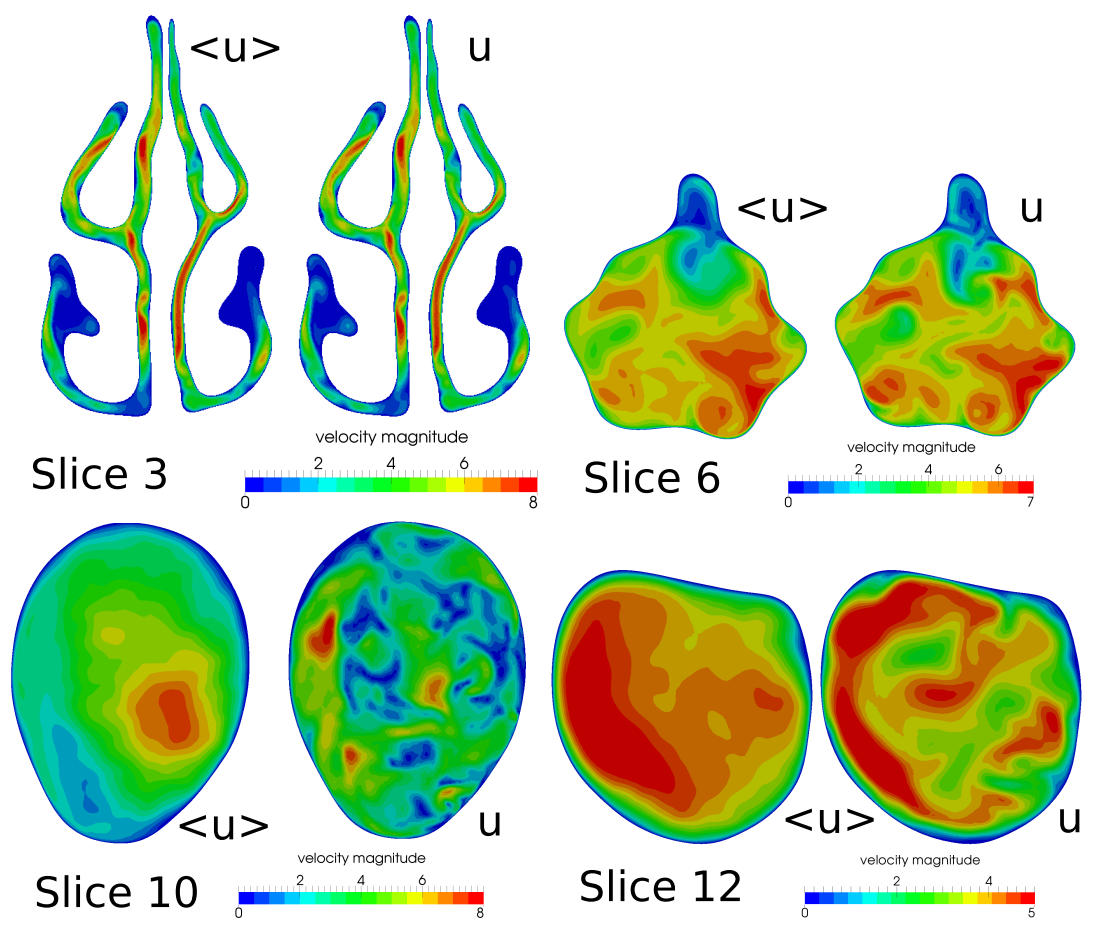

Figure 8: Different cross-sectional slices of the airways, referenced in Figure 2, $\langle\mathbf{u}\rangle$ the time average velocity (computed for the time period [0.1-0.15 s]), and $\mathbf{u}$ the instantaneous velocity $(0.15 \mathrm{~s})$. 

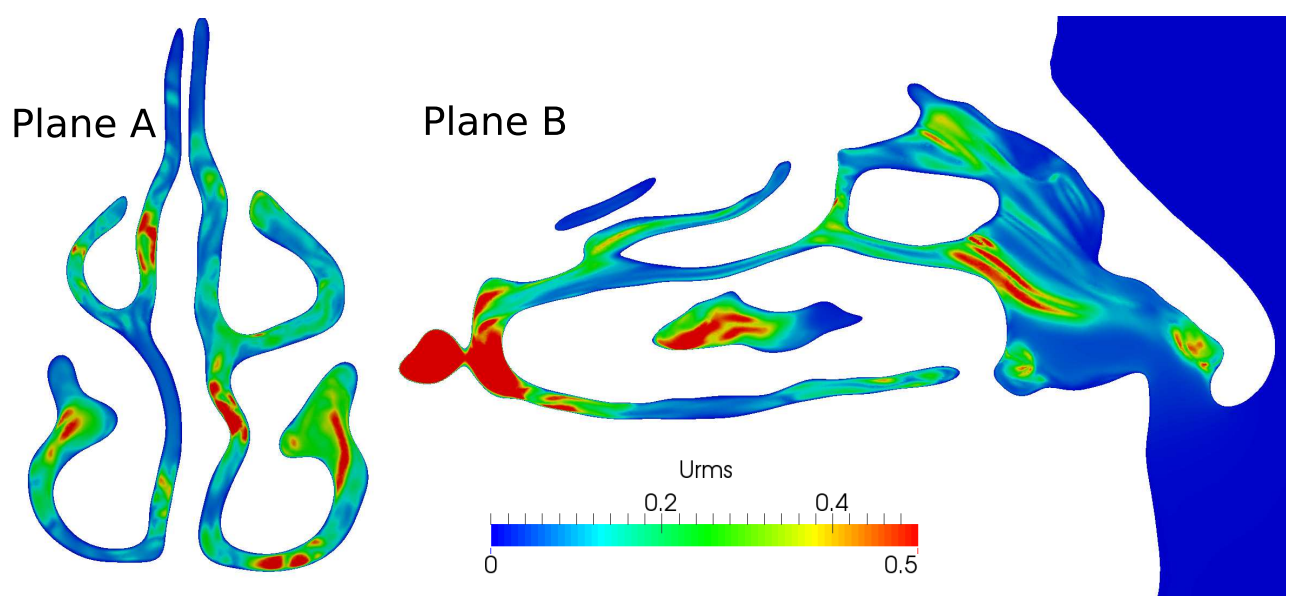

(a)

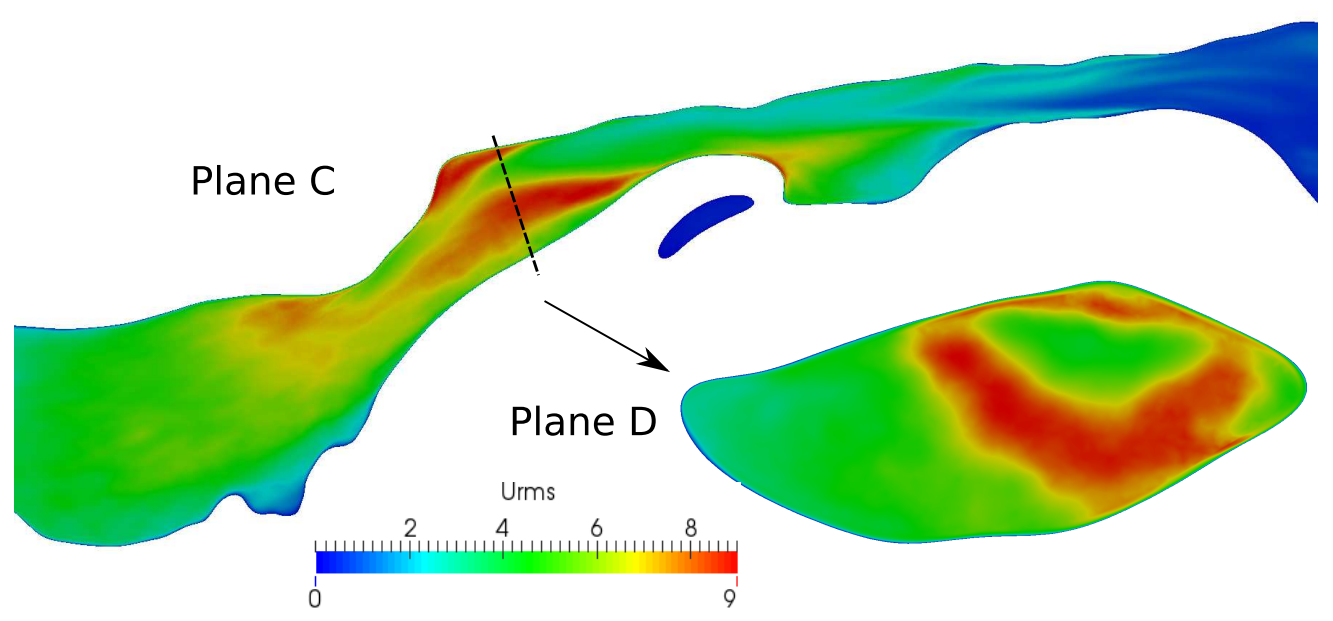

(b)

Figure 9: rms velocity fluctuations magnitude of a few cross-sections. (a) Coronal plane in the middle of nasal cavity (plane A) and sagittal plane of right nasal cavity (plane B). (b) Sagittal plane of laryngotracheal area (plane $\mathrm{C}$ ) and a coronal plane of the supra-glottic region(plane D). (a) and (b) are computed for the time period [0.1-0.15 s] and the rms velocity fluctuations scale is different to enhance the features in the nasal cavity and in the laryngotracheal area. See Figure 2 for cross-section location. 
in the present study, the flow in the right nasal cavity is more unsteady than in the left nasal cavity. On comparing the cross-sections of mean and fluctuating velocity in the nasal cavity, shown respectively in Figures 6 and 9, the regions of higher $\left|\mathbf{u}_{r m s}^{\prime}\right|$ do not correlate with the regions of higher mean velocity, but instead these regions occur at the edges of high velocity regions, indicating that the unsteadiness is caused by the breakdown of shear layers.

Conversely, in the laryngeal region cross-sections shown in Figures 6 and 9, the regions of high $\left|\mathbf{u}_{r m s}^{\prime}\right|$ correspond to the shear layers located on the boundary of the laryngeal jet, indicating that in this region the fluctuations in the velocity are likely related to a high Reynolds number. The high values of $\left|\mathbf{u}_{r m s}^{\prime}\right|$ persist downstream of the laryngeal stenosis where the jet is initiated, into the trachea with decreasing magnitude and localization. These findings are related to the turbulent dissipation through smaller scales, which occurs rapidly in the laryngeal region due to the change in direction of the airway that forces the jet to impinge on the posterior glottis region, and thus favour vortex breakdown.

\subsection{Wall-shear stress}

The wall-shear stress is an important measure for the respiratory airways, being responsible to a large extent for the resistive forces during respiration, and related to exchange processes and the near-wall flow field [33]. In Figure 10 the surface integrated wall-shear stress for different compartments (see Figure 2) of the airways are plotted. The thin and scroll-like form of the nasal cavities exposes the flow to a large wall surface area, such that despite having, in general, low wall-shear stress values, the integrated stress (between sections 2-4, see Figure 11 for slice location map) is greater than the other regions. A large resistance also occurs between sections 9-12, which correspond to the passages of the hypopharynx and further downstream. However, in contrast, the wallshear stress values in these regions are large and the wetted surface area is in comparison small. On the whole, compartmentalising the anatomy, the greatest resistance is reported between section 6-12, which corresponds to the descending airways from the oropharynx. The nasal valve (present between section 1-2) also 

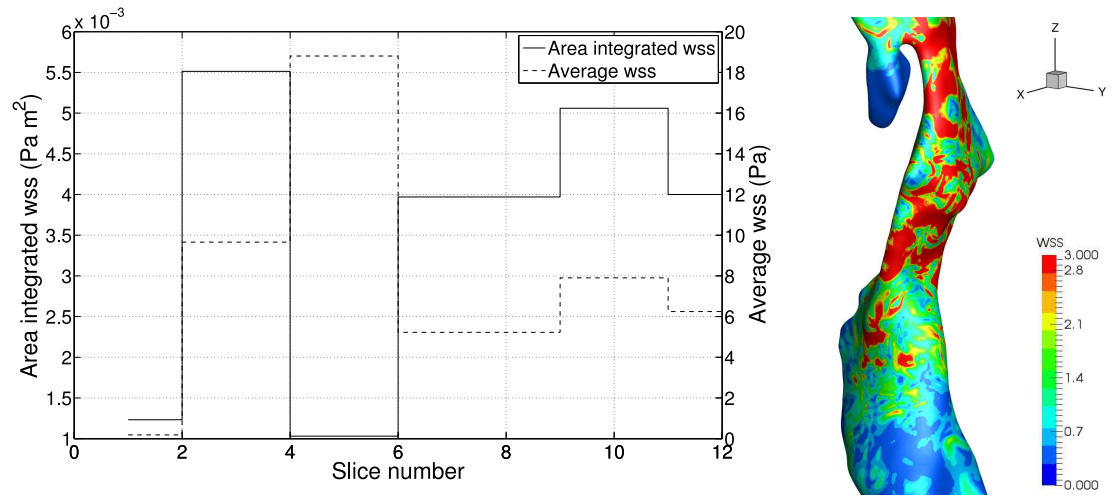

(a)

(b)

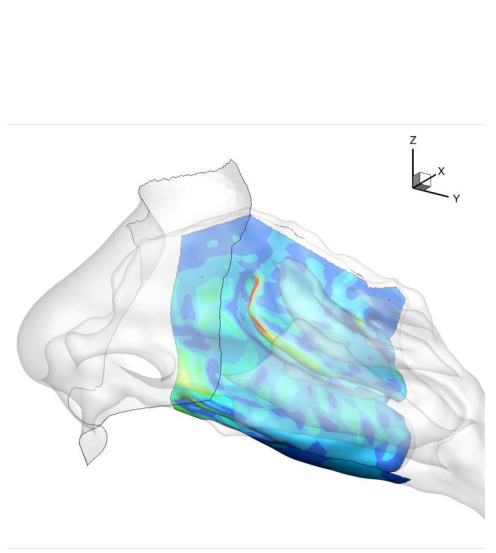

(c)

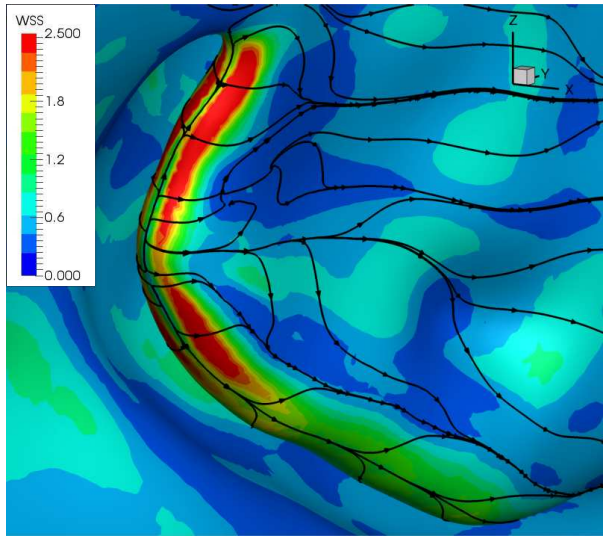

(d)

Figure 10: (a) The surface integrated wall-shear stress value over different sections on the left hand vertical axis and the average wall-shear stress (i.e. the surface integrated wall-shear stress divided by the considered area surface) on the right hand vertical axis (see Figure 11). (b) Map of wall-shear stress magnitude in the hypopharynx and trachea (sections 8-11). (c) General view of map of wall-shear stress magnitude in the right nasal cavity mid-region. (d) Detail of the anterior protrusion of the right nasal cavity middle turbinate, with map of wallshear stress magnitude and surface shear lines. All results correspond to results at the time $\mathrm{t}=0.15 \mathrm{~s}$. 
causes considerable resistance due to the constriction.

The complex, undulating shape of the wall at the mid-region of the nasal cavity affects the flow by partitioning it into different regions. The undulations may also favour exposing core flow to the nasal lining, enhancing exchange processes between lining and the flow as well as deposition of suspended particles. The turbinates are important geometric features in the partitioning of the flow, and in Figure 10 a detail of the anterior protrusion of the middle turbinate is shown, with the wall-shear stress magnitude and the surface shear lines (computed as integrated paths of the wall shear stress). As noted above in Section 3.2, the nasal valve forms a jet that is directed at the anterior protrusion of the middle turbinate, and as it impinges a stagnation line is clearly visible at the leading edge. Furthermore, the surface shear lines show that the flow is directed away on either side of this impingement line.

The wall-shear stress, surface shear lines, and consequently the near-wall flow field [33], vary smoothly in the nasal cavity. These findings are in contrast to the region of the hypopharynx where a bend in the conduit is present, and we observe a higher and disturbed wall-shear stress magnitude, as shown in Figure 10. The impingement zone in the hypopharynx breaks down the flow, enhancing mixing and bringing the core flow in contact with the lining. This highly energetic zone of impingement and mixing may serve to enhance deposition of smaller particles still in suspension that were not filtered in the nasal cavity.

\subsection{Energy flux}

In order to understand the physiological aspects of flow resistance during breathing, we plot the energy flux along the respiratory tract in Figure 11. The energy flux is the rate of transfer of energy across a surface, $\Gamma$, and is given by:

$$
\text { Energy flux }=E=\int_{\Gamma}\left[\left(P+\frac{1}{2} \rho|\mathbf{u}|^{2}\right)|\mathbf{u}|\right] d \Gamma
$$

where $P$ is static pressure, $\frac{1}{2} \rho|\mathbf{u}|^{2}$ is dynamic pressure, $\rho$ the density of the air and $|\mathbf{u}|$ the velocity magnitude of the airflow. Time averaged measures were 

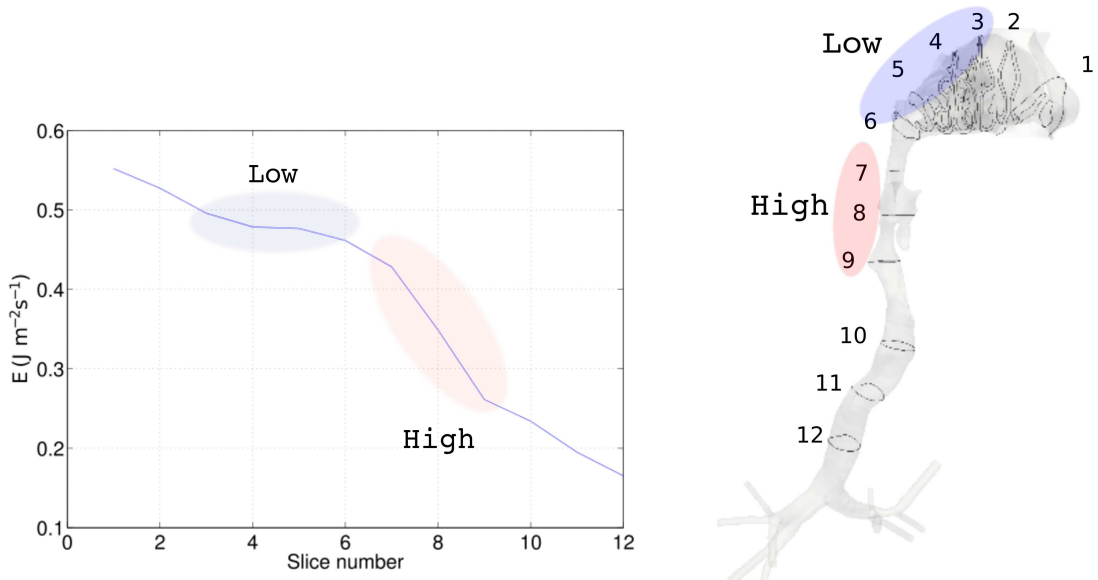

Figure 11: Energy flux computed at different sections along the airways.

used for the period $[0.1-0.15 s]$. The energy flux is computed at different cross-sections in the domain, with an orientation approximately normal to the mean flow.

As expected, the energy flux decreases along the tract at varying rates. The sharpest decreases in energy flux are coincident to large values in the integrated wall-shear stress (Figure 10), indicating that the wall-shear stress plays a key role in the resistive forces during a sniff. We observe that in a few regions the energy flux does not decrease noticeably, and these correspond to regions where the flow enters a larger airspace and comparatively slows down, noticeably in the nasopharynx and the lower portion of the trachea.

\subsection{Flapping and transitional flow in the right nasal cavity}

In the following sections the unsteady dynamics of the flow are considered. The flow in the nasal cavity is transitional just downstream of the nasal valve due to the jet that is formed, but is remarkably steady after the anterior meatuses due to the reduced calibre of the passages and the large surface area. The lower airways, however, in the regions of the larynx and the trachea, exhibit turbulent flow that is triggered by the laryngeal jet and the curvature of the passage.

In order to fully resolve the temporal and spatial scales directly, the results of 
this section are computed using the finest mesh (M3). To provide reproducibility and clarity of results, the spectral analysis in this work is performed with a shorttime Fourier transform, with a width equal to half the period and an overlap of $50 \%$ in order to reduce signal noise, and with a Hann window to ensure periodicity.

The main body of the nasal valve jet effectively does not exhibit oscillatory motion. A key feature of the transitional flow downstream of the nasal valve is the vortex shedding and flapping motion of the shear layer, located on the boundary between the nasal valve jet and the superior separated flow region. Transient motion is also seen on the lower boundary of the jet but to a smaller extent. The velocity and vorticity magnitude are plotted in Figure 12 for a sagittal plane cross-section of the right nasal cavity, together with the spectral analysis of the velocity at two locations for the time period [0.08-0.12 s].

The instabilities initiate at the rapid change in geometry, downstream of the nasal valve, and are amplified as they advect downstream. As such, the oscillations recorded at the upstream point (3B) occur shortly before being observed at the downstream point $(3 \mathrm{~A})$, with a delay of $\sim 0.002 \mathrm{~s}$. This delay is calculated by observing the recorded time lag of the first main peak of the oscillations, for points $3 \mathrm{~A}$ and $3 \mathrm{~B}$, and fits with the observation that the time average velocity magnitude in this region $\langle|\mathbf{u}|\rangle \approx 5 \mathrm{~m} / \mathrm{s}$ and the distance between $3 \mathrm{~A}$ and $3 \mathrm{~B}$ is $\approx 0.01 \mathrm{~m}$. The flapping of the shear layer observed between points $3 \mathrm{~B}$ and $3 \mathrm{~A}$, induces the roll-up and subsequent shedding of vortices, as shown in Figure 13.

The amplitude of the flapping motion increases downstream, being larger at point $3 \mathrm{~A}$ than at point $3 \mathrm{~B}$. From the spectral analysis of the velocity magnitude calculated for the time window [0.08-0.12 s], the dominant frequency modes and amplitudes are rather dissimilar at these two points. There is a noticeable decrease in the modal energy and dominant frequency of oscillation for the upstream point (3B) with respect to the downstream point (3A). The dominant frequency recorded at location $3 \mathrm{~A}(350 \mathrm{~Hz})$ is approximately three times greater in amplitude than that recorded at location 3B. However, point locations do not represent the entire 3D field. 


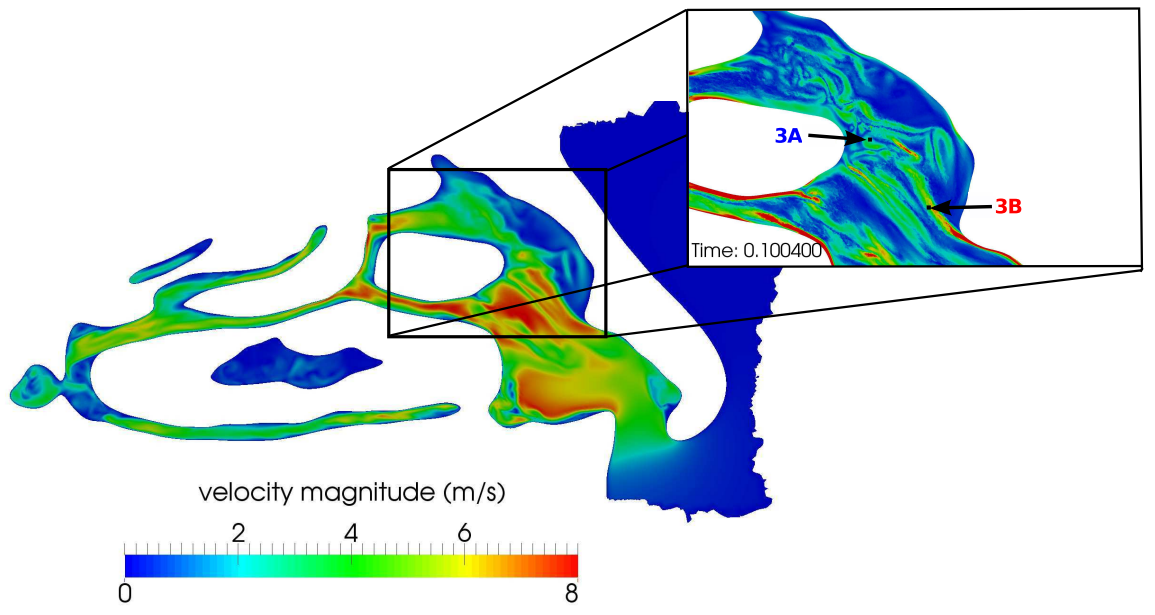

(a)

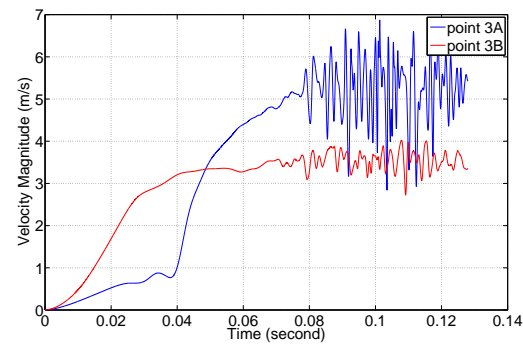

(b)

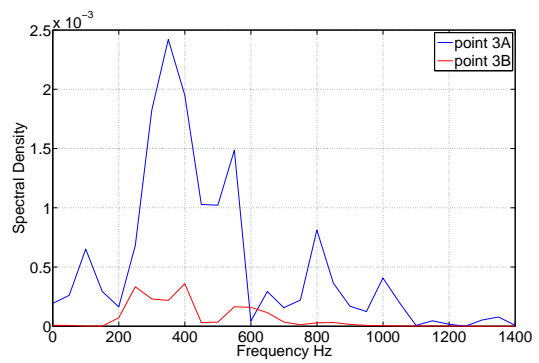

(c)

Figure 12: (a) Cross-section in the sagittal plane of the right nasal cavity (Figure 2, plane B), showing the velocity magnitude and vorticity magnitude (the zoom highlights the occurrence of the flapping between 3A and 3B). (b) Temporal evolution and (c) spectral analysis of the velocity magnitude at two different locations (3A and $3 \mathrm{~B})$, during the beginning of the sniff waveform. 


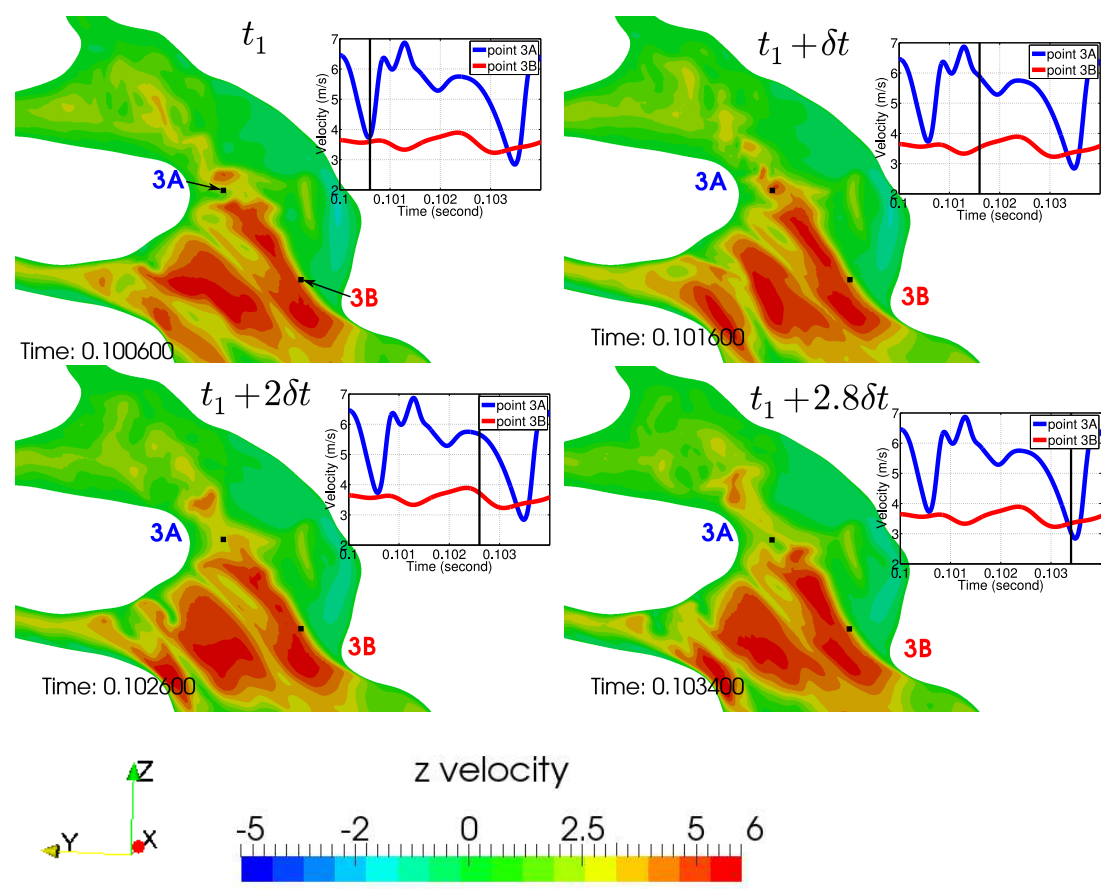

Figure 13: Four snapshots in the sagittal plane of the right nasal cavity, showing the vertical component of the velocity. Traces of the temporal evolution of the velocity magnitude recorded at two locations are also shown, where the vertical line indicates the current snapshot time.

The development of the vortex shedding in the upper and anterior right nasal cavity is affected by the interaction with the geometry. The narrowness of the cavity dissipates the velocity gradients and the flow structures, while also increasing the vorticity magnitude. Snapshots during the vortex shedding discussed above are shown in Figure 13. The period of the vortex shedding is approximately $0.0028 \mathrm{~s}$, or a frequency equal to $350 \mathrm{~Hz}$. It is the dominant frequency recorded at location 3A, as show in the spectral analysis in Figure 12.

Unlike the right nasal cavity, the left does not exhibit a flow separation region superior to the nasal valve jet. Despite this difference, the flow remains unsteady in the left cavity and transitional during the plateau phase of the sniff waveform. A greater analysis of the bilateral cavities will be the scope of subsequent investigation. 


\subsection{Turbulent flow in the laryngeal and trachea regions}

We will now concentrate on the analysis of the flow field along the descending airways. The dominant turbulent flow is observed in the descending airways due to the laryngeal jet. The anatomy of the subject harbours a marked reduction in airway area above the glottis due to the supine position maintained during the CT scan. In this posture the tongue and soft tissues have lowered and reduced the normal patency of the airways. A strong jet is observed that, together with the varying shape of the airways, results in a turbulent flow field that quickly dissipates through the breakup of the vortex structures.

A one dimensional spectral analysis, that considers only the vertical component of the velocity fluctuation is computed for the time period [0.1-0.15 s], the start of the plateau phase of the sniff waveform. Only the vertical component of the velocity is considered because it is approximately the streamwise direction. Here the spectrum amplitude $\left(E_{11}\right)$ is normalised by the Kolmogorov length scale $\eta=\left(\nu^{3} / \epsilon\right)^{1 / 4}$, where $\epsilon=2 \nu\left\langle S_{i j} S_{i j}\right\rangle$ is the dissipation rate and $S_{i j}$ is the strain rate tensor. Here, four overlapping Hann window functions are used for the short-time Fourier transform to reduce noise. The result at three points located within the laryngeal jet as it enters the trachea, are presented in Figure 14. These three locations were chosen to lie downstream of the laryngeal jet where the greatest velocity magnitude is observed, seen schematically in the figure by the mean velocity iso-surface (see also Figure 7 for detail).

In this work, Taylor's hypothesis assumption is used. We convert the time sampled signal into spatial sampling with a bulk flow advection, where $\kappa=$ $2 \pi f /\langle|\mathbf{u}|\rangle$ is the wave number related to the sampling frequency $f$ and $\langle|\mathbf{u}|\rangle$ the local time average velocity magnitude. Varghese et al. [13] recommends the use of characteristics of the constriction to normalize the energy spectra, however here the Kolmogorov scaling is used to normalize the energy spectra following Pope [34] and Saddoughi et al. [35], for convenience and comparison purposes.

As such we can investigate the tracheal turbulent dissipation. The lines that appear in the figures correspond to the $-5 / 3$ slope, which is known as the inertial sub-range and is associated with the range of frequencies in which the energy 


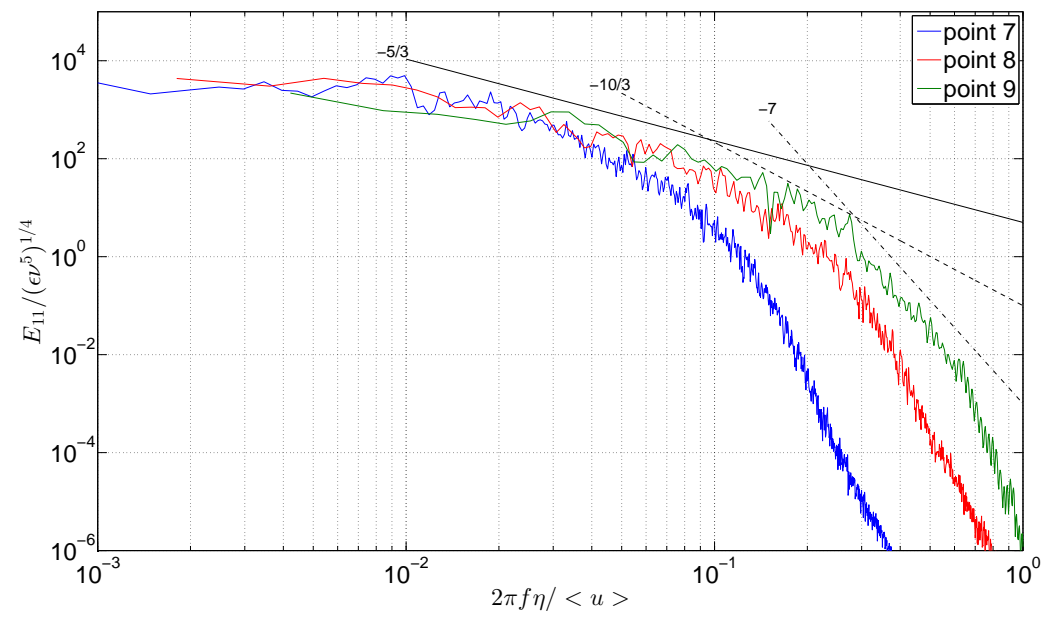

(a)

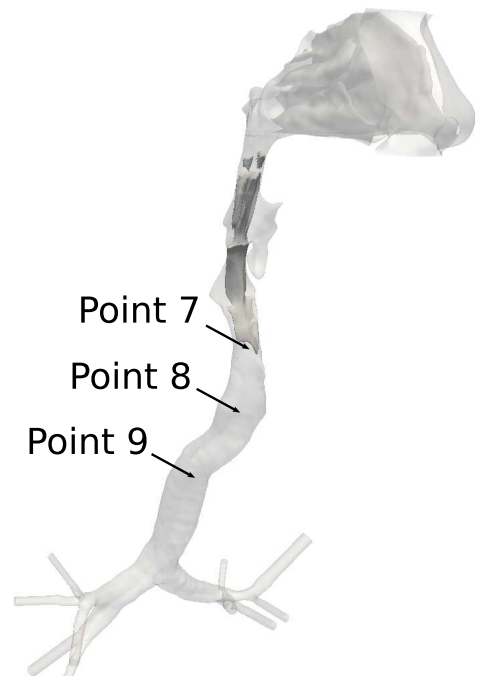

(b)

Figure 14: (a) Normalized energy spectrum of streamwise velocity fluctuations at three different locations downstream of the jet. (b) The laryngeal jet and the location of the three points. 


\begin{tabular}{|l|c|c|c|}
\hline & Point 7 & Point 8 & Point 9 \\
\hline$A\left(m^{2}\right) * 10^{-4}$ & 1.5 & 2.2 & 2.0 \\
\hline$\langle|\mathbf{u}|\rangle(m / s)$ & 16.5 & 6 & 3.6 \\
\hline$R e * 10^{5}$ & 2.2 & 1.0 & 0.5 \\
\hline$\epsilon\left(m^{3} / s^{-3}\right)$ & 3300 & 1100 & 250 \\
\hline$\eta(\mu m)$ & 32 & 42 & 60 \\
\hline
\end{tabular}

Table 2: Local informations about the time average velocity magnitude $(\langle|\mathbf{u}|\rangle)$, the cross sectional area $(A)$, the Reynolds Number based of the hydraulic diameter $(R e)$, the dissipation rate $(\epsilon)$ and the kolmogorov lenght scale $(\eta)$ in the three locations of picture 14 .

cascade takes place. In this region, dominated by inertial transfer, the energy production is equal to the energy dissipation. The $-10 / 3$ slope represents the range of frequencies characteristic of the post-stenotic flow $[12,13,36]$. The -7 slope characterizes the dissipation range, where viscous forces dominate[34].

The energy contained at the low frequency on the 3 different spectra are approximately equal. However, a few distinct peaks in the low frequency portion of the spectrum are observed at location 7 , which are not observed at locations 8 and 9 . These low frequency modes are due to the presence of larger structures produced by the laryngeal jet created by the upstream constriction.

The inertial sub-range, represented by the $-5 / 3$ slope, is seen to widen and cover a greater portion of the spectrum as the flow progresses downstream. This observation indicates that progressing downstream from the region of turbulence production (the laryngeal stenosis in this subject), the energy transfers from larger to smaller eddy size, and consequently occupies a larger range at higher frequencies. These observations are consistent with the work of Varghese et al. [13] which suggest that after a certain distance from the constriction, fully developed turbulent flow is obtained for an idealised geometry. In the case of this specific subject, highly non-equilibrium flow is observed approximately around the start of the trachea.

As expected and agreeing with the observations of Varghese et al. [13], the post-stenotic regime (-10/3 slope) is more evident closer to the constriction at 
location 7 , while this regime decreases further downstream at locations 8 and 9 .

In Table 2 , the time average velocity magnitude $(\langle|\mathbf{u}|\rangle)$, the cross sectional area $(A)$, the Reynolds number $(R e)$ based of the hydraulic diameter, the time average velocity magnitude and the kinetic viscosity of air, the dissipation rate $(\epsilon)$ and the Kolmogorov length scale $(\eta)$ are reported for the three locations identified in Figure 14 during the time period [0.1-0.15 s] of the sniff waveform. As we progress downstream of the jet, both mean velocity magnitude and dissipation rate decrease, while the cross-sectional area is approximately constant. The higher velocity fluctuations, which are related to the dissipation rate, decrease as the flow moves downstream and was described also by Varghese et al. [13]. With a decrease in dissipation rate, the Kolmogorov length scale increases as the flow enters the smaller airways.

The values of the Kolmogorov length scale $\eta$, are of the same order as those observed in a pipe flow with the Reynolds number $R e \approx 10^{5}$, see [37]. The implication of this observation is that the complex morphology of the conduit does not play an important role in terms of the turbulence intensity. Studies on idealised pipe flow geometries may therefore be sufficient to represent dominant features of the real flow.

Figure 15 shows the ratio of $\mathbf{u}_{r m s}^{\prime} /\langle|\mathbf{u}|\rangle$ computed at different points along the descending airway. This ratio is defined as turbulence level or turbulence intensity. There is a high turbulence zone for the points 8 and $9(\approx 40 \%)$. After these points, the turbulence level decreases quickly as the flow advects downstream, with a value at the carina (point 12) equal to $10 \%$. The value of the turbulence intensity at one of the second level bifurcation (point 13) is $5 \%$.

\subsection{Energy spectra}

A spectral analysis of the turbulent kinetic energy is computed for the time period [0.1-0.13 s], the start of the plateau phase of the sniff waveform. Turbulent kinetic energy is defined as:

$$
T K E=0.5\left(\left\langle u^{\prime 2}\right\rangle+\left\langle v^{\prime 2}\right\rangle+\left\langle w^{\prime 2}\right\rangle\right) \quad \text { with } \quad \mathbf{u}^{\prime}=\mathbf{u}-\langle\mathbf{u}\rangle
$$




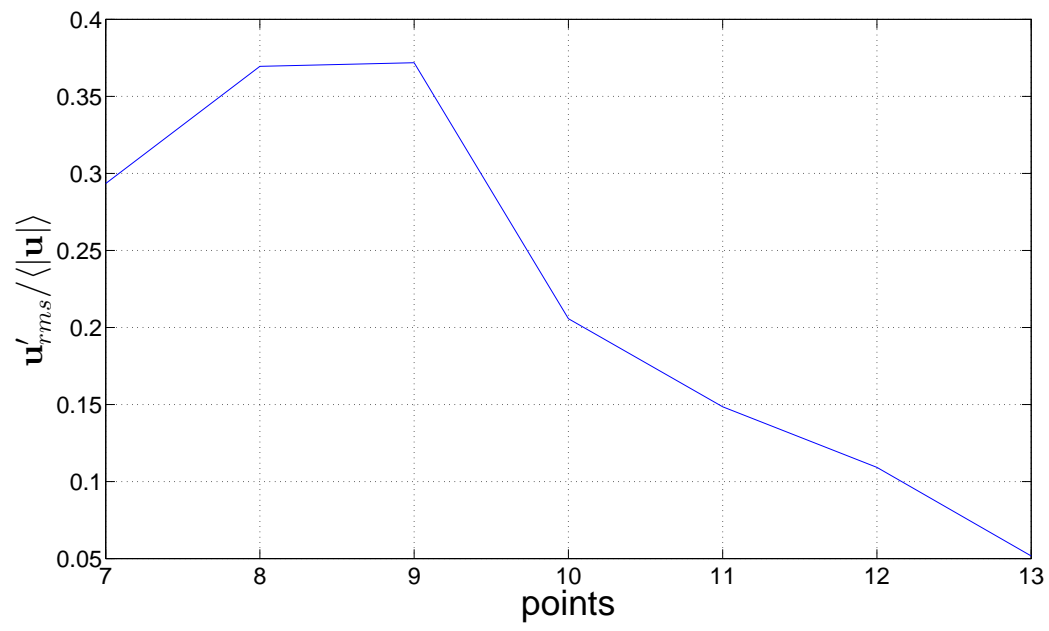

(a)

Point 7 .

Point 8

Point 9

Point 10

Point 11

Point 12

Point 13

(b)

Figure 15: (a) Ratio of $\mathbf{u}_{r m s}^{\prime} /\langle|\mathbf{u}|\rangle$ computed at different points along the descending airway until the lung. (b) Representation of these point locations. 
where $\langle\mathbf{u}\rangle$ denotes the time average velocity, $\mathbf{u}^{\prime}$ is the fluctuation of the velocity, and $\mathbf{u}$ is the instantaneous velocity signal.

Here the energy spectra is not normalized in the frequency domain, and is presented in Figure 16 for four locations at two mesh resolutions. In order to compare the spectra, we conserve the same scaling. The four locations exhibit dramatically different flow features: the right nasal cavity (point 3), the oropharynx (point 5), the larynx (point 7), and the trachea (point 9) (locations referenced also in Figure 2). These locations were chosen to investigate the turbulence characteristics occurring along the tract.

Figure 16 shows that the energy content is markedly different at the different locations, with the spectral energy content in the larynx (point 7) being the highest due to the presence of large scale structures produced by the laryngeal jet. It can also be observed that the lowest energy content is located in the right nasal cavity (point 3), which is consistent with the analysis of root mean square velocity fluctuations, discussed above in Section 3.3. The well-defined low frequency peaks observed at point 3, are related to the vortex shedding as discussed in Section 3.6. The spectra scaling of the point 3 is not well adapted to the signal but as mentioned above, in order to compare the spectra, we conserve the same scaling.

As the inspired air traverses the upper airways, it first enters the nasal cavity where the turbulence intensity is low, as similarly reported by Zhao et al. [1]. On reaching the oropharynx, turbulence is produced given the increasing velocity due to smaller cross-sectional areas. Considering the spectral energy content, the highest turbulence intensity is observed in the larynx and is related to the presence of the large structures. In the larynx, the inertial sub-range (-5/3 slope) extends to a wide frequency range compared to the other locations $\left(1 \times 10^{3}-5 \times 10^{3} \mathrm{~Hz}\right)$. The $-10 / 3$ slope associated with the post-stenotic flow is also more evident at point 7 . As the flow descends the trachea, the energy of the large structures is dissipated, hence the energy content decreases and the vortical structures are broken down into smaller ones. As a consequence, the frequency spectrum and the ranges are of reduced size in comparison to the 


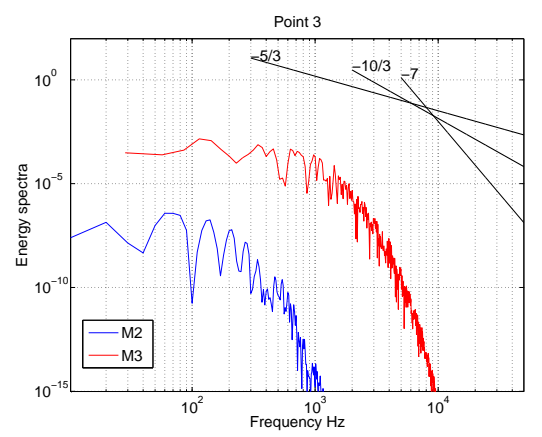

(a)

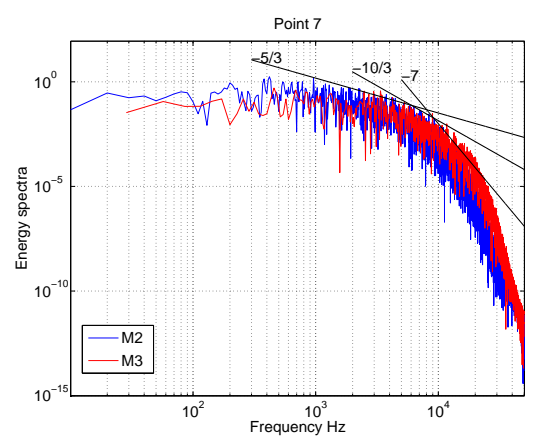

(c)

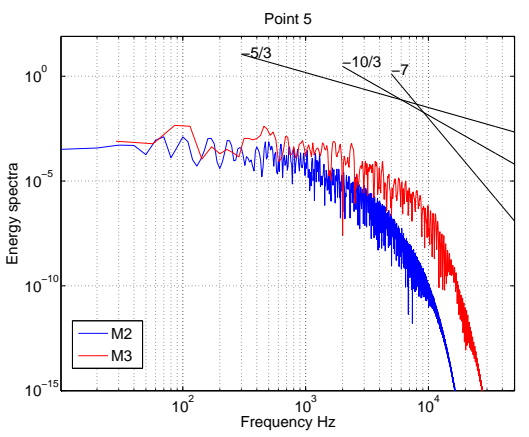

(b)

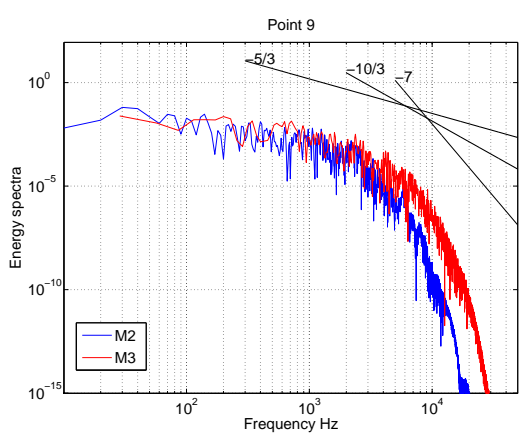

(d)

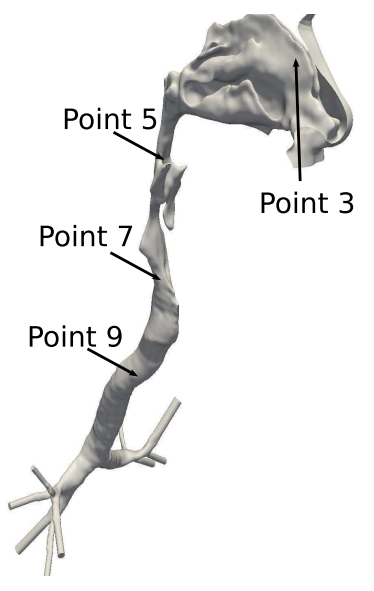

(e)

Figure 16: Energy spectra in four different locations, three lines denote the slopes of $-5 / 3$, $-10 / 3$ and -7 , as noticed on the spectrum figures. (a) Point 3. (b) Point 5. (c) Point 7. (d) point 9. (e) Location of the points 
findings at point 7 .

For both mesh resolutions, the inertial sub-range is similar and can be considered to be well resolved. The effect of a finer mesh resolution is seen however for the post-stenotic flow range, which extends further at higher frequencies for the finer mesh (M3). Another observation is that the slope -7 develops at lower frequencies for the M2 mesh $(44 \mathrm{M})$, at $10^{4} \mathrm{~Hz}$, than the M3 mesh, which is approximately at $1.5 \times 10^{4} \mathrm{~Hz}$. The -7 slope concerns the dominance of viscous forces.

\section{Conclusions and discussion}

The purpose of this study was to explore the dynamics of unsteady flow in the large airways during a rapid inhalation through large-scale CFD on a highly-detailed geometry. Rapid inhalations are of relevance to sniffing for both olfaction and drug delivery of aerosols. The flow-rate considered (peak of $900 \mathrm{mLs}^{-1}$ ) corresponds to breathing at a somewhat elevated level but well below the transition from nasal to oro-nasal breathing. Resolving such flows poses a computational challenge and few previous attempts have been made to fully resolve the dynamics.

The results presented here were obtained on a series of progressively refined computational meshes, the finest one containing 350 million elements.

These simulations show that, despite the highly unsteady nature of the flow, the dominant features are established quite rapidly.

It was also found that spontaneous fluctuations in nasal airflow velocities occur, resulting from shear layer instabilities (jet breakdown, flapping). The capture of these required the finest mesh resolution. However the impact of these features on the fluctuation intensity was relatively low.

High levels of wall-shear stress were observed where flow impacts on the nasal turbinates and in the confined space of the supra-glottic airway.

Fourier spectral analysis showed that the spectrum of flow fluctuations in the nose is consistent with a transitional but not fully turbulent flow, being of 
limited bandwidth.

The dominant pressure loss was associated with the laryngeal jet. The comparison of the pressure drop is very similar for 350 and 44 million elements but the mean pressure loss for the 44 million case is higher than that for the 350 million case.

The peak Reynolds number based on the effective diameter for the jet computed at point 7 is equal to $2.2 \times 10^{5}$, which is in the turbulent regime. The spectral analysis demonstrates similarities to fully developed turbulence.

The fluctuation level is decreasing as the flow progresses further downstream from point 9 in the trachea; approximately $10 \mathrm{~cm}$ appear sufficient to remove most fluctuations from the flow entering the bronchi. As a future project, we will apply the same large-scale CFD simulation strategy going further, including the bronchioles.

In summary, it has been found that whilst spontaneous fluctuations in velocity arise in the nose, the dominant site of turbulence production during inhalation is in the supra-glottic region, as opposed to the glottis itself. This finding is likely related to the effect of posture on the subject airway and highlights the need for further investigative studies. High-fidelity CFD simulations revealed the causes of flow unsteadiness and enabled the production and decay of turbulence measures to be mapped. These results show the potential of largescale CFD simulations on detailed patient-specific geometries as a benchmark for future investigations.

\section{Acknowledgements}

We acknowledge PRACE for awarding us access to resource FERMI based in Italy at Bologna hosted by Cineca. This work was financially supported by the PRACE project Pra04 693. (2011050693 to the Fourth PRACE regular call). The second author gratefully acknowledges support from project MatComPhys under the European Research Executive Agency FP7-PEOPLE2011-IEF framework. The third author was supported by the Engineering and 
Physical Sciences Research Council [grant number EP/M506345/1].

\section{Appendix}

Point coordinates. The list of points used in this study, is given in the table 3 . The points are normalised by the tip of the nose which here is considered to be the point 1.

\begin{tabular}{|l|c|c|c|}
\hline Points & Coordinate $\mathrm{X}(m)$ & Coordinate $\mathrm{Y}(m)$ & Coordinate $\mathrm{Z}(m)$ \\
\hline 1 & 0.0 & 0.0 & 0.0 \\
\hline 2 & -0.1060 & 0.0484 & 0.0090 \\
\hline 3 & -0.0086 & 0.0487 & 0.0196 \\
\hline $3 \mathrm{~A}$ & -0.0073 & 0.0564 & 0.0273 \\
\hline $3 \mathrm{~B}$ & -0.0084 & 0.0501 & 0.0210 \\
\hline 4 & -0.0082 & 0.1256 & 0.0061 \\
\hline 5 & -0.0023 & 0.1379 & -0.0104 \\
\hline 7 & 0.0009 & 0.1500 & -0.0704 \\
\hline 8 & 0.0013 & 0.1437 & -0.1123 \\
\hline 9 & -0.0061 & 0.1553 & -0.1304 \\
\hline 10 & -0.0028 & 0.1735 & -0.1504 \\
\hline 11 & -0.0052 & 0.1867 & -0.1704 \\
\hline 12 & -0.0068 & 0.2024 & -0.2046 \\
\hline 13 & -0.0394 & 0.2069 & -0.2046 \\
\hline
\end{tabular}

Table 3: Coordinates of the points in Figure 2 relative to the tip of nose.

Modelling of the sniff. To model the sniff inflow, we use a polynomial function of order 10 derived from the experimental work detailed in Rennie et al. [30]. The $10^{t h}$ order polynomial function describing the temporal evolution of the flow rate is provided in Figure 3. 


\begin{tabular}{|l|c|}
\hline Order & Coefficient \\
\hline \hline 0 & 0.0015 \\
\hline 1 & 0.6813 \\
\hline 2 & 472.2648 \\
\hline 3 & -10790.8421 \\
\hline 4 & 114269.4525 \\
\hline 5 & 705483.2425 \\
\hline 6 & 2736179.6777 \\
\hline 7 & -6773661.0554 \\
\hline 8 & 10395525.1742 \\
\hline 9 & -9007136.2292 \\
\hline 10 & 3364786.7418 \\
\hline
\end{tabular}

Table 4: Coefficient values of the $10^{\text {th }}$ order polynomial function

Medical case.A short description of the medical case is given bellow. A consultant radiologist reported the nasal airways as clear and of normal appearance. The position of the tongue base and other soft tissues in the pharynx were deemed consistent with the patient being scanned in the supine position. The vocal cords were noted to have the appearance of being abducted, whereas the trachea was considered to be of normal dimensions, not demonstrating any abnormalities. The airway in the pharynx may be narrower than if the patient had been standing, but the geometry is within the normal range.

[1] K. Zhao, P. Dalton, G. C. Yang, P. W. Scherer, Numerical modeling of turbulent and laminar airflow and odorant transport during sniffing in the human and rat nose, Chemical senses 31 (2006) 107-118.

[2] D. J. Doorly, D. J. Taylor, A. M. Gambaruto, R. C. Schroter, N. Tolley, Nasal architecture: Form and flow, Phil. Trans. Roy. Soc. A 366 (2008) $3225-3246$. 
[3] D. J. Doorly, D. J. Taylor, R. C. Schroter, Mechanics of airflow in the human nasal airways, Resp. Physiol. Neurobiol. 163 (2008) 100-110.

[4] A. Lintermann, M. Meinke, W. Schröder, Fluid mechanics based classification of the respiratory efficiency of several nasal cavities, Computers in biology and medicine 43 (2013) 1833-1852.

[5] D. J. Taylor, D. J. Doorly, R. C. Schroter, Inflow boundary profile prescription for numerical simulation of nasal airflow, Journal of the Royal Society Interface 7 (2010) 515-527.

[6] S. T. Jayaraju, M. Brouns, C. Lacor, B. Belkassem, S. Verbanck, Large eddy and detached eddy simulations of fluid flow and particle deposition in a human mouth-throat, Journal of Aerosol Science 39 (2008) 862-875.

[7] C. G. Ball, M. Uddin, A. Pollard, Mean flow structures inside the human upper airway, Flow, Turbulence and Combustion 81 (2008) 155-188.

[8] E. Ghahramani, O. Abouali, H. Emdad, G. Ahmadi, Numerical analysis of stochastic dispersion of micro-particles in turbulent flows in a realistic model of human nasal/upper airway, Journal of Aerosol Science 67 (2014) 188-206.

[9] P. H. Saksono, P. Nithiarasu, I. Sazonov, S. Y. Yeo, Computational flow studies in a subject-specific human upper airway using a one-equation turbulence model. influence of the nasal cavity, International Journal for $\mathrm{Nu}-$ merical Methods in Engineering 87 (2011) 96-114.

[10] C. L. Lin, M. H. Tawhai, G. McLennan, E. A. Hoffman, Characteristics of the turbulent laryngeal jet and its effect on airflow in the human intrathoracic airways, Respiratory physiology \& neurobiology 157 (2007) 295309.

[11] D. Elad, S. Naftali, M. Rosenfeld, M. Wolf, Physical stresses at the air-wall interface of the human nasal cavity during breathing, Journal of Applied Physiology 100 (2006) 1003-1010. 
[12] J. Choi, M. H. Tawhai, E. A. Hoffman, C. L. Lin, On inter- and intrasubject variabilities of airflow in the human lungs, Physics of Fluids 21 (2009).

[13] S. S. Varghese, S. H. Frankel, P. Fischer, Direct numerical simulation of stenotic flows. part 1. steady flow, Journal of Fluid Mechanics 582 (2007) $253-280$.

[14] B. A. Craven, E. G. Paterson, G. S. Settles, M. J. Lawson, Development and verification of a high-fidelity computational fluid dynamics model of canine nasal airflow, Journal of biomechanical engineering 131 (2009) 091002.

[15] A. J. Bates, D. J. Doorly, R. Cetto, H. Calmet, A. M. Gambaruto, N. S. Tolley, G. Houzeaux, R. C. Schroter, Dynamics of airflow in a short inhalationand, J. R. Soc. Interface (2015).

[16] G. Taubin, Curve and surface smoothing without shrinkage, in: Computer Vision, 1995. Proceedings., Fifth International Conference on, IEEE, 1995, pp. $852-857$.

[17] D. A. Field, Laplacian smoothing and delaunay triangulations, Communications in applied numerical methods 4 (1988) 709-712.

[18] ANSYS Meshing User's Guide, 2000. http://148.204.81.206/Ansys/ 150/ANSYSMeshingUsersGuide.pdf.

[19] S. K. Robinson, Coherent motions in the turbulent boundary layer, Annual Review of Fluid Mechanics 23 (1991) 601-639.

[20] U. Piomelli, E. Balaras, Wall-layer models for large-eddy simulations, Annual review of fluid mechanics 34 (2002) 349-374.

[21] G. Houzeaux, R. de la Cruz, H. Owen, M. Vázquez, Parallel uniform mesh multiplication applied to a navier-stokes solver, Computers \& Fluids 80 (2013) 142-151. 
[22] Alya System, Large Scale Computational Mechanics, 2000. http://www . bsc.es/computer-applications/alya-system.

[23] C. O, S. Badia, R. Codina, J. Principe, Assessment of variational multiscale models for the large eddy simulation of turbulent incompressible flows, Computer Methods in Applied Mechanics and Engineering 285 (2015) 3263.

[24] G. Houzeaux, J. Principe, A variational subgrid scale model for transient incompressible flows, IJCFD 22 (2008) 135-152.

[25] G. Houzeaux, R. Aubry, M. Vázquez, Extension of fractional step techniques for incompressible flows: The preconditioned orthomin(1) for the pressure schur complement, Computers \& Fluids 44 (2011) 297-313.

[26] R. Lohner, F. Mut, J. Cebral, R. Aubry, G. Houzeaux, Deflated preconditioned conjugate gradient solvers for the pressure-poisson equation: Extensions and improvements, Int. J. Numer. Meth. Engn. 87 (2011) 2-14.

[27] O. Soto, R. Löhner, F. Camelli, A linelet preconditioner for incompressible flow solvers, Int. J. Num. Meth. Heat Fluid Flow 13 (2003) 133-147.

[28] G. Karypis, Metis: Serial graph partitioning and fill-reducing matrix ordering, 1995-2015. Available at: http://glaros.dtc.umn.edu/gkhome/ views/metis.

[29] M. Folk, A. Cheng, K. Yates, Hdf5: A file format and i/o library for high performance computing applications, in: Proceedings of Supercomputing, volume 99, 1999, pp. 5-33.

[30] C. E. Rennie, K. A. Gouder, D. J. Taylor, N. S. Tolley, R. C. Schroter, D. J. Doorly., Nasal inspiratory flow: at rest and sniffing., Int Forum Allergy Rhinol 1 (2011) 128-135.

[31] A. M. Gambaruto, D. J. Taylor, D. J. Doorly, Modelling nasal airflow using a fourier descriptor representation of geometry, IJNMF 59 (2009) 1259-1283. 
[32] S. T. Jayaraju, M. Brouns, S. Verbanck, C. Lacor, Fluid flow and particle deposition analysis in a realistic extrathoracic airway model using unstructured grids, Journal of Aerosol Science 38 (2007) 494-508.

[33] A. M. Gambaruto, D. J. Doorly, T. Yamaguchi, Wall shear stress and near-wall convective transport: Comparisons with vascular remodelling in a peripheral graft anastomosis, Journal of Computational Physics 229 (2010) $5339-5356$.

[34] S. B. Pope, Turbulent flows, Cambridge university press, 2000.

[35] S. G. Saddoughi, S. V. Veeravalli, Local isotropy in turbulent boundary layers at high reynolds number, Journal of Fluid Mechanics 268 (1994) $333-372$.

[36] M. K. Bang, H. C. William, Experimental measurements of turbulence spectra distal to stenoses, Journal of Biomechanics 7 (1974) 335 - 342.

[37] J. Tu, K. Inthavong, G. Ahmadi, Computational fluid and particle dynamics in the human respiratory system, Springer, 2012. 